CRYSTALLOGRAPHIC COMMUNICATIONS

ISSN 2056-9890

Received 11 April 2016

Accepted 19 April 2016

Edited by A. J. Lough, University of Toronto, Canada

Keywords: crystal structure; brucinium salts; $p$ arsanilic acid; water stabilization; hydrogen bonding.

CCDC reference: 1475231

Supporting information: this article has supporting information at journals.iucr.org/e

\section{Crystal structure and hydrogen bonding in the water-stabilized proton-transfer salt brucinium 4-aminophenylarsonate tetrahydrate}

\author{
Graham Smith* and Urs D. Wermuth
}

Science and Engineering Faculty, Queensland University of Technology, GPO Box 2434, Brisbane, Queensland 4001, Australia. *Correspondence e-mail: g.smith@qut.edu.au

In the structure of the brucinium salt of 4-aminophenylarsonic acid ( $p$-arsanilic acid), systematically 2,3-dimethoxy-10-oxostrychnidinium 4-aminophenylarsonate tetrahydrate, $\left(\mathrm{C}_{23} \mathrm{H}_{27} \mathrm{~N}_{2} \mathrm{O}_{4}\right)\left[\mathrm{As}\left(\mathrm{C}_{6} \mathrm{H}_{7} \mathrm{~N}\right) \mathrm{O}_{2}(\mathrm{OH})\right] \cdot 4 \mathrm{H}_{2} \mathrm{O}$, the brucinium cations form the characteristic undulating and overlapping head-to-tail layered brucine substructures packed along [010]. The arsanilate anions and the water molecules of solvation are accommodated between the layers and are linked to them through a primary cation $\mathrm{N}-\mathrm{H} \cdots \mathrm{O}$ (anion) hydrogen bond, as well as through water $\mathrm{O}-\mathrm{H}$... O hydrogen bonds to brucinium and arsanilate ions as well as bridging water $\mathrm{O}$-atom acceptors, giving an overall three-dimensional network structure.

\section{Chemical context}

The Strychnos alkaloid base brucine, (2,3-dimethoxystrychnidin-10-one; BRU) has been extensively employed as a resolving agent for chiral organic compounds (Wilen, 1972). With chiral acids, the separation is achieved through protontransfer to N19 of the strychnidine cage $\left(\mathrm{p} K_{\mathrm{a} 2}=11.7\right.$; O'Neil, 2001), followed by separation of the resultant crystalline salt products by fractional crystallization. Similar effects are achieved with the essentially identical Strychnos alkaloid strychnine but separation efficiency favours brucine. This is probably because of the formation in the crystal of characteristic brucinium host substructures comprising head-to-tail undulating layers of brucine molecules or cations which accommodate selectively the hydrogen-bonded guest molecules in the crystal structure. A characteristic of the substructure is the repeat interval in the layer of $c a 12.3 \AA$ along a $2_{1}$ screw axis in the crystal, which is reflected in the unit-cell dimension, with brucine being predominantly in the monoclinic space group $P 2_{1}$ or the orthorhombic space group $P 22_{1} 2_{1}$ (Smith, Wermuth \& White, 2006; Smith, Wermuth, Young \& White, 2006).

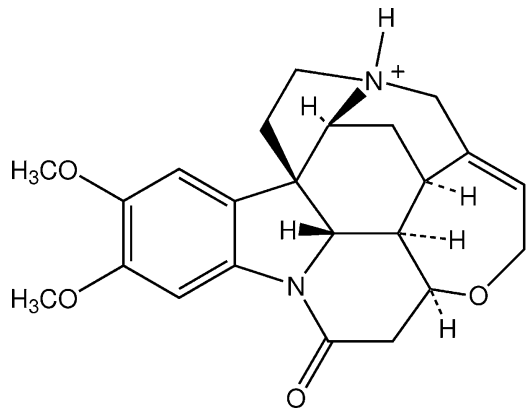<smiles>Nc1ccc([As](=O)(O)O)cc1</smiles>

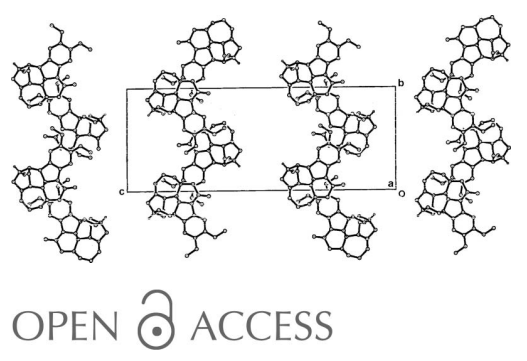


This example of molecular recognition was described in the early structure determinations of brucinium benzoyl-Dalaninate (Gould \& Walkinshaw, 1984) and in the structures of the pseudopolymorphic brucine solvates, brucine- $\mathrm{MeOH}$ (1:1) and brucine-EtOH-water (1/1/2) (Glover et al., 1985). The guest molecules are accommodated interstitially within the layers and are commonly accompanied by compatible polar solvent molecules, usually generating high-dimensional hydrogen-bonded crystal structures.

Currently, a large number of structures of brucine compounds with chiral organic molecules, including both acids and non-acids are known, but in addition those with achiral compounds also feature. Of interest to us have been the structures of brucinium proton-transfer salts with largely simple organic acids, prepared under aqueous alcoholic conditions, the crystalline products being stabilized by solvent molecules. Water-stabilized achiral carboxylate examples include $\mathrm{BRU}^{+}$hydrogen fumarate ${ }^{-} \cdot 1.5 \mathrm{H}_{2} \mathrm{O}$ (Dijksma, Gould, Parsons \& Walkinshaw, 1998), $\mathrm{BRU}^{+}$dihydrogen citrate ${ }^{-}$.$3 \mathrm{H}_{2} \mathrm{O}$ (Smith, Wermuth \& White, 2005) and $\mathrm{BRU}^{+}$benzoate $^{-} \cdot 3 \mathrm{H}_{2} \mathrm{O}$ (Białońska \& Ciunik, 2006b).

Other organic acids besides carboxylates may be included among the set but fewer structural examples are known, e.g. sulfonates $\left(\mathrm{BRU}^{+}\right.$toluene-4-sulfonate ${ }^{-} \cdot 3 \mathrm{H}_{2} \mathrm{O}$; Smith, Wermuth, Healy et al., 2005). However, no brucinium arsonate structures are known, so that the reaction of brucine with 4-aminophenylarsonic acid (p-arsanilic acid) in 2-propanol/ water was carried out, resulting in the formation of the crystalline hydrated title salt, $\mathrm{C}_{23} \mathrm{H}_{27} \mathrm{~N}_{2} \mathrm{O}_{4}{ }^{+} \cdot \mathrm{C}_{6} \mathrm{H}_{7} \mathrm{AsNO}_{3}{ }^{-} \cdot 4 \mathrm{H}_{2} \mathrm{O}$, and the structure is reported herein. The acid has biological significance as an anti-helminth in veterinary applications (Thomas, 1905; Steverding, 2010) and as a monohydrated sodium salt (atoxyl) which had early usage as an anti-syphilitic (Ehrlich \& Bertheim, 1907; Bosch \& Rosich, 2008). Simple $p$-arsanilate salt structures are not common in the Cambridge Structural Database (Groom et al., 2016), with only the $\mathrm{NH}_{4}{ }^{+}$
Table 1

Hydrogen-bond geometry $\left(\AA{ }^{\circ}\right)$.

\begin{tabular}{lllll}
\hline$D-\mathrm{H} \cdots A$ & $D-\mathrm{H}$ & $\mathrm{H} \cdots A$ & $D \cdots A$ & $D-\mathrm{H} \cdots A$ \\
\hline $\mathrm{N} 19-\mathrm{H} 19 \cdots \mathrm{O} 12 A$ & $0.91(4)$ & $1.72(4)$ & $2.610(3)$ & $168(4)$ \\
$\mathrm{N} 4 A-\mathrm{H} 41 A \cdots \mathrm{O} 4 W^{\mathrm{i}}$ & $0.89(3)$ & $2.46(4)$ & $3.291(5)$ & $155(4)$ \\
$\mathrm{N} 4 A-\mathrm{H} 42 A \cdots \mathrm{O} 3 W^{\mathrm{ii}}$ & $0.90(3)$ & $2.25(3)$ & $3.137(6)$ & $169(4)$ \\
$\mathrm{O} 13 A-\mathrm{H} 13 A \cdots \mathrm{O} 11 A^{\mathrm{ii}}$ & $0.90(4)$ & $1.67(4)$ & $2.546(3)$ & $165(4)$ \\
$\mathrm{O} 1 W-\mathrm{H} 11 W \cdots \mathrm{O} 25$ & $0.90(4)$ & $1.95(4)$ & $2.843(4)$ & $175(3)$ \\
$\mathrm{O} 1 W-\mathrm{H} 12 W \cdots \mathrm{O} 2 W^{\mathrm{iii}}$ & $0.90(3)$ & $1.87(4)$ & $2.760(5)$ & $168(4)$ \\
$\mathrm{O} 2 W-\mathrm{H} 21 W \cdots \mathrm{O} 12 A$ & $0.90(3)$ & $2.11(3)$ & $2.945(4)$ & $153(4)$ \\
$\mathrm{O} 2 W-\mathrm{H} 22 W \cdots \mathrm{O} 11 A^{\text {iv }}$ & $0.89(3)$ & $2.07(4)$ & $2.915(4)$ & $158(5)$ \\
$\mathrm{O} 3 W-\mathrm{H} 31 W \cdots \mathrm{O} 25^{\mathrm{v}}$ & $0.91(4)$ & $2.06(4)$ & $2.922(4)$ & $159(3)$ \\
$\mathrm{O} 3 W-\mathrm{H} 32 W \cdots \mathrm{O} 4 W^{\text {vi }}$ & $0.91(3)$ & $1.91(3)$ & $2.791(4)$ & $164(3)$ \\
$\mathrm{O} 4 W-\mathrm{H} 41 W \cdots \mathrm{O} 1 W^{\mathrm{vii}}$ & $0.90(4)$ & $1.88(4)$ & $2.770(5)$ & $172(5)$ \\
$\mathrm{O} 4 W-\mathrm{H} 42 W \cdots \mathrm{O} 12 A$ & $0.89(4)$ & $1.91(4)$ & $2.802(4)$ & $174(5)$ \\
$\mathrm{C} 14-\mathrm{H} 14 \cdots \mathrm{O} 3^{\text {viii }}$ & 1.00 & 2.52 & $3.363(4)$ & 142 \\
$\mathrm{C} 15-\mathrm{H} 151 \cdots \mathrm{O} 11 A^{\text {ii }}$ & 0.99 & 2.60 & $3.561(4)$ & 165 \\
$\mathrm{C} 18-\mathrm{H} 182 \cdots \mathrm{O} 2 W$ & 0.99 & 2.58 & $3.422(5)$ & 143 \\
$\mathrm{C} 20-\mathrm{H} 201 \cdots \mathrm{O} 11 A^{\text {ii }}$ & 0.99 & 2.41 & $3.388(4)$ & 170 \\
$\mathrm{C} 20-\mathrm{H} 202 \cdots \mathrm{O} 13 A^{\text {iv }}$ & 0.99 & 2.43 & $3.229(4)$ & 137
\end{tabular}

Symmetry codes: (i) $\quad x-\frac{1}{2},-y+\frac{3}{2},-z+1$; (ii) $\quad x+\frac{1}{2},-y+\frac{1}{2},-z+1$; (iii) $-x, y-\frac{1}{2},-z+\frac{1}{2} ; \quad$ (iv) $\quad x-\frac{1}{2},-y+\frac{1}{2},-z+1 ; \quad$ (v) $-x+\frac{1}{2},-y+1, z+\frac{1}{2}$; $\quad$ (vi) $x+\frac{1}{2},-y+\frac{3}{2},-z+1$; (vii) $-x+1, y+\frac{1}{2},-z+\frac{1}{2}$; (viii) $-x+1, y-\frac{1}{2},-z+\frac{1}{2}$.

and $\mathrm{K}^{+}$salts (Smith \& Wermuth, 2014) and the guanidinium salts (Smith \& Wermuth, 2010; Latham et al., 2011) being known.

\section{Structural commentary}

The asymmetric unit of the title salt comprises a brucinium cation, a $p$-arsanilate anion $A$ and four water molecules of solvation, $(\mathrm{O} 1 \mathrm{~W}-\mathrm{O} 4 \mathrm{~W})$, all inter-associated through hydrogen bonds (Fig. 1). Protonation has occurred as expected at N19 of the brucine cage, the invoked Peerdeman (1956) absolute configuration for the strychnidinium molecule giving the overall Cahn-Ingold stereochemistry of the cation as C7 $(R)$, $\mathrm{C} 8(S), \mathrm{C} 12(S), \mathrm{C} 13(R), \mathrm{C} 14(R), \mathrm{C} 16(S)$ and the additional introduced $(S)$ chiral centre at N19.

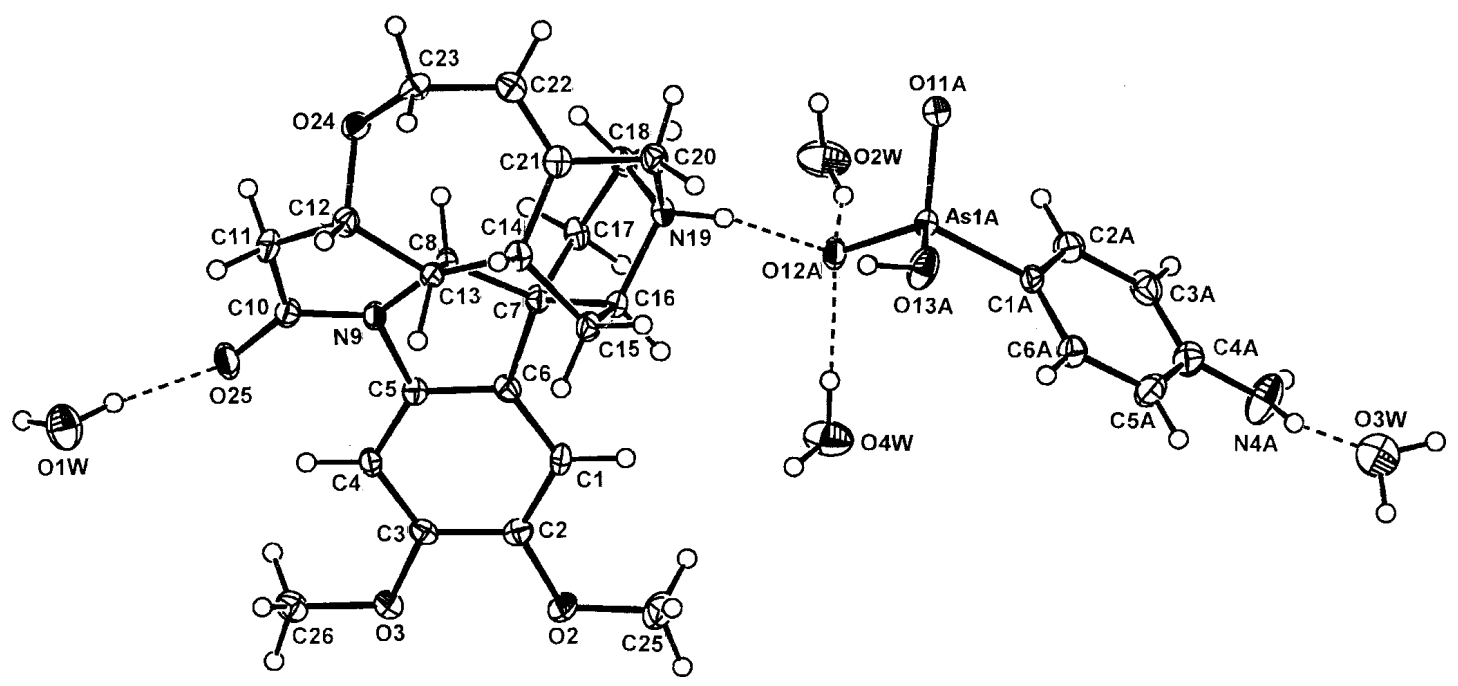

Figure 1

Molecular configuration and atom-numbering scheme for the brucinium cation, $p$-arsanilate anion $A$ and the four water molecules of solvation in the asymmetric unit of the title salt. Inter-species hydrogen bonds are shown as dashed lines. Non-H atoms are shown as $40 \%$ probability displacement ellipsoids. 


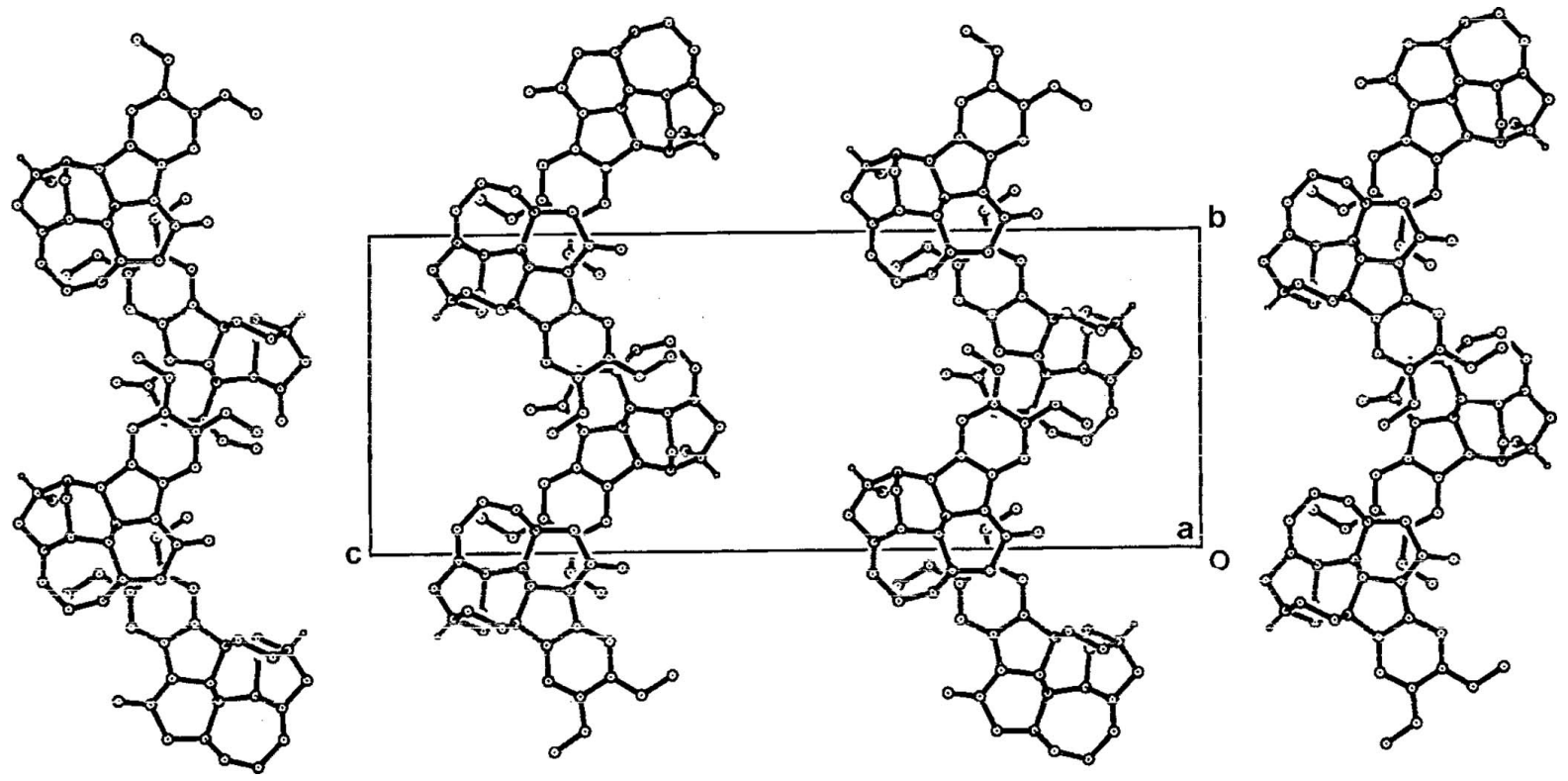

Figure 2

The undulating brucinium sheet substructures in the unit cell of the title salt, less the inter-sheet anion and water molecules, viewed down $a$. All $\mathrm{H}$ atoms except that of the protonated N19 atom have also been removed.

\section{Supramolecular features}

The brucinium cations form into the previously described undulating sheet-host substructures which are considered to be the reason for the molecular recognition peculiar to brucine (Gould \& Walkinshaw, 1984; Gould et al., 1985; Dijksma, Gould, Parsons \& Walkinshaw, 1998; Dijksma, Gould, Parsons, Taylor \& Walkinshaw, 1998; Oshikawa et al., 2002; Białońska \& Ciunik, 2004). In the title salt, these substructures extend along the $b$-axis direction, with the previously described 2 propagation of the brucinium cations along the $c a 12.3 \AA$ axis (Fig. 2). The $p$-arsanilate anions and the water molecules occupy the interstitial spaces in the structure. The protonated N19 atom of the cation gives a single hydrogen-bonding interaction with a $p$-arsanilate oxygen acceptor $(\mathrm{O} 12 A)$ while two of the solvent water molecules $(\mathrm{O} 1 W$ and $\mathrm{O} 3 W$ ) form hydrogen bonds with the

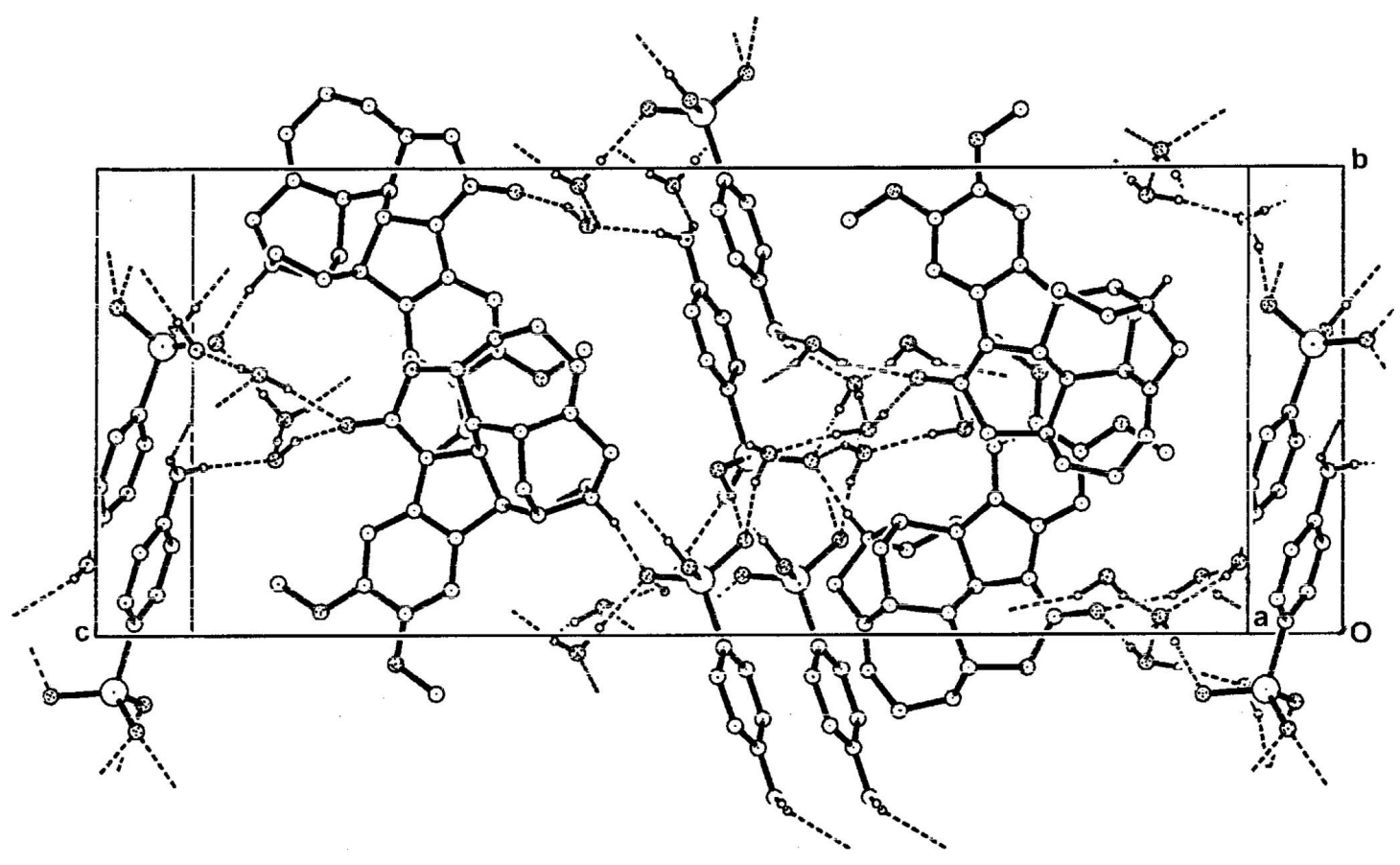

Figure 3

A perspective view of the packing in the unit cell, viewed along the approximate $a$-axial direction, showing the associated anions and the water molecules in the interstitial regions of the brucinium layered substructures, with hydrogen-bonding interactions shown as dashed lines. 
carbonyl $\mathrm{O} 25$ atom of the the brucinium cation (Table 1). Within the inter-sheet channels, the $p$-arsanilate anions are linked head-to-head through an $\mathrm{O} 13 A-\mathrm{H} \cdots \mathrm{O} 11 A^{\mathrm{ii}}$ hydrogen bond while both $\mathrm{H}$ atoms of the amine group form hydrogen bonds with water molecules $\mathrm{O} 3 W$ and $\mathrm{O} 4 W^{\mathrm{i}}$. The water molecules $\mathrm{O} 2 W$ and $\mathrm{O} 4 A$ are further linked to the $p$-arsanilate $\mathrm{O}$-atom $\mathrm{O} 12 A$ with $\mathrm{O} 2 W$ also linked to $\mathrm{O} 11 A^{\mathrm{iv}}$. Water molecules $\mathrm{O} 3 W$ and $\mathrm{O} 4 W^{\mathrm{i}}$ give inter-water hydrogen bonds and together with a number of inter-molecular $\mathrm{C}-\mathrm{H} \cdots \mathrm{O}$ interactions (Table 1) result in an overall three-dimensional network structure (Fig. 3).

\section{Database survey}

Interstitial water molecules are present in the structures of the brucine pseudo-polymorphic structures, e.g. the common tetrahydrate form and the 5.2 hydrate (Smith et al., 2006a) and the dihydrate (Smith et al., 2007), as well as the mixed solvates BRU-EtOH- $\mathrm{H}_{2} \mathrm{O}(1 / 1 / 2)$ (Glover et al., 1985) and BRU-i$\mathrm{PrOH}-\mathrm{H}_{2} \mathrm{O}$ (1/1/2) (Białońska \& Ciunik, 2004). A large number of water-stabilized brucinium salts of acids are known: with the inorganic sulfate $(\mathrm{BRU})_{2} \mathrm{SO}_{4} \cdot 7 \mathrm{H}_{2} \mathrm{O}$ (Białońska \& Ciunik, 2005) and most commonly with aromatic carboxylates, e.g. the benzoate (a trihydrate; Białońska \& Ciunik, 2006b); the 4-nitrobenzoate (a dihydrate; Białońska \& Ciunik, 2007); the 3,5-dinitrobenzoate (a trihydrate; Białońska \& Ciunik, 2006a); the 3,5-dinitrosalicylate (a monohydrate; Smith et al., 2006a); the phthalate (a monohydrate; Krishnan, Gayathri, Sivakumar, Gunasekaran \& Anbalagen, 2013); the hydrogen isophthalate (a trihydrate; Smith, Wermuth, Young \& White, 2006); the hydrogen 3-nitrophthalate (a dihydrate; Smith, Wermuth, Young \& Healy, 2005) and the picraminobenzoate (a monohydrate; Smith \& Wermuth, 2011).

Aliphatic carboxylate examples are: with hydrogen oxalate (a dihydrate; Krishnan, Gayathri, Sivakumar, Chakkaravathi \& Anbalagen, 2013); with hydrogen fumarate (a sesquihydrate; Dijksma, Gould, Parsons \& Walkinshaw, 1998); with hydrogen $(S)$-malate (a pentahydrate; Smith, Wermuth \& White, 2006); with dihydrogen citrate (a trihydrate; Smith, Wermuth \& White, 2005); with L-glycerate (a 4.75 hydrate; Białońska et al., 2005) and with hydrogen cis-cyclohexane-1,2dicarboxylate (a dihydrate; Smith et al., 2012). Some sulfonate salts are also known, e.g. with toluene-4-sulfonate (a trihydrate; Smith, Wermuth, Healy et al., 2005); with 3-carboxy-4hydroxybenzenesulfonate (a pentahydrate; Smith et al., $2006 b$ ) and with biphenyl-4,4'-disulfonate (a hexahydrate; Smith et al., 2010).

\section{Synthesis and crystallization}

The title compound was synthesized by heating together under reflux for $10 \mathrm{~min}, 1 \mathrm{mmol}$ quantities of brucine tetrahydrate and 4-aminophenylarsonic acid in $50 \mathrm{~mL}$ of $80 \%$ 2-propanol/ water. After concentration to $c a 30 \mathrm{~mL}$, partial roomtemperature evaporation of the hot-filtered solution gave thin colourless crystal plates of the title compound from which a specimen was cleaved for the X-ray analysis.
Table 2

Experimental details.

\begin{tabular}{|c|c|}
\hline \multicolumn{2}{|l|}{ Crystal data } \\
\hline Chemical formula & $\begin{array}{c}\left(\mathrm{C}_{23} \mathrm{H}_{27} \mathrm{~N}_{2} \mathrm{O}_{4}\right)\left[\mathrm{As}\left(\mathrm{C}_{6} \mathrm{H}_{7-}\right.\right. \\
\left.\mathrm{N}) \mathrm{O}_{2}(\mathrm{OH})\right] \cdot 4 \mathrm{H}_{2} \mathrm{O}\end{array}$ \\
\hline$M_{\mathrm{r}}$ & 683.58 \\
\hline Crystal system, space group & Orthorhombic, $P 2_{1} 2_{1} 2_{1}$ \\
\hline Temperature $(\mathrm{K})$ & 200 \\
\hline$a, b, c(\AA)$ & $7.6553(3), 12.3238(5), 31.960(2)$ \\
\hline$V\left(\AA^{3}\right)$ & $3015.2(3)$ \\
\hline$Z$ & 4 \\
\hline Radiation type & Mo $K \alpha$ \\
\hline$\mu\left(\mathrm{mm}^{-1}\right)$ & 1.19 \\
\hline Crystal size $(\mathrm{mm})$ & $0.36 \times 0.34 \times 0.10$ \\
\hline \multicolumn{2}{|l|}{ Data collection } \\
\hline Diffractometer & $\begin{array}{l}\text { Oxford Diffraction Gemini-S } \\
\text { CCD-detector diffractometer }\end{array}$ \\
\hline Absorption correction & $\begin{array}{l}\text { Multi-scan (CrysAlis PRO; Rigaku } \\
\text { OD, 2015) }\end{array}$ \\
\hline$T_{\min }, T_{\max }$ & $0.811,0.980$ \\
\hline $\begin{array}{l}\text { No. of measured, independent and } \\
\text { observed }[I>2 \sigma(I)] \text { reflections }\end{array}$ & $11983,6980,5901$ \\
\hline$R_{\mathrm{int}}$ & 0.032 \\
\hline$(\sin \theta / \lambda)_{\max }\left(\AA^{-1}\right)$ & 0.693 \\
\hline \multicolumn{2}{|l|}{ Refinement } \\
\hline$R\left[F^{2}>2 \sigma\left(F^{2}\right)\right], w R\left(F^{2}\right), S$ & $0.048,0.096,1.05$ \\
\hline No. of reflections & 6980 \\
\hline No. of parameters & 433 \\
\hline No. of restraints & 14 \\
\hline $\mathrm{H}$-atom treatment & $\begin{array}{l}\mathrm{H} \text { atoms treated by a mixture of } \\
\text { independent and constrained } \\
\text { refinement }\end{array}$ \\
\hline$\Delta \rho_{\max }, \Delta \rho_{\min }\left(\mathrm{e} \AA^{-3}\right)$ & $0.55,-0.46$ \\
\hline Absolute structure & Flack (1983), 3672 Friedel pairs \\
\hline Absolute structure parameter & $-0.005(9)$ \\
\hline
\end{tabular}

Computer programs: CrysAlis PRO (Rigaku OD, 2015), SIR92 (Altomare et al., 1993), SHELXL97 (Sheldrick, 2008) within WinGX (Farrugia, 2012) and PLATON (Spek, 2009).

\section{Refinement details}

Crystal data, data collection and structure refinement details are summarized in Table 2. Hydrogen atoms potentially involved in hydrogen-bonding interactions were located by difference methods but their positional parameters were constrained in the refinement with $\mathrm{N}-\mathrm{H}$ and $\mathrm{O}-\mathrm{H}=0.90 \AA$, and with $U_{\text {iso }}(\mathrm{H})=1.2 U_{\text {eq }}(\mathrm{N})$ or $1.5 U_{\text {eq }}(\mathrm{O})$. Other $\mathrm{H}$ atoms were included in the refinement at calculated positions [C$\mathrm{H}($ aromatic $)=0.95 \AA$ and $\mathrm{C}-\mathrm{H}$ (aliphatic) $=0.97-1.00 \AA]$ and treated as riding with $U_{\text {iso }}(\mathrm{H})=1.2 U_{\text {eq }}(\mathrm{C})$. The absolute configuration determined for the parent strychnidinin-10-one molecule (Peerdeman, 1956) was invoked and was confirmed in the the structure refinement.

\section{Acknowledgements}

The authors acknowledge support from the Science and Engineering Faculty, Queensland University of Technology.

\section{References}

Altomare, A., Cascarano, G., Giacovazzo, C. \& Guagliardi, A. (1993). J. Appl. Cryst. 26, 343-350. 
Białońska, A. \& Ciunik, Z. (2004). Acta Cryst. C60, o853-o855.

Białońska, A. \& Ciunik, Z. (2005). Acta Cryst. E61, o4222-o4224.

Białońska, A. \& Ciunik, Z. (2006a). Acta Cryst. C62, o450-o453.

Białońska, A. \& Ciunik, Z. (2006b). Acta Cryst. E62, o5817-o5819.

Białońska, A. \& Ciunik, Z. (2007). Acta Cryst. C63, o120-o122.

Białońska, A., Ciunik, Z., Popek, T. \& Lis, T. (2005). Acta Cryst. C61, o88-091.

Bosch, F. \& Rosich, L. (2008). Pharmacology, 82, 171-179.

Dijksma, F. J. J., Gould, R. O., Parsons, S., Taylor, J. \& Walkinshaw, M. D. (1998). Chem. Commun. pp. 745-746.

Dijksma, F. J. J., Gould, R. O., Parsons, S. \& Walkinshaw, M. D. (1998). Acta Cryst. C54, 1948-1951.

Ehrlich, P. \& Bertheim, A. (1907). Berichte, pp. 3292-3297.

Farrugia, L. J. (2012). J. Appl. Cryst. 45, 849-854.

Flack, H. D. (1983). Acta Cryst. A39, 876-881.

Glover, S. S. B., Gould, R. O. \& Walkinshaw, M. D. (1985). Acta Cryst. C41, 990-994.

Gould, R. O., Kelly, R. \& Walkinshaw, M. D. (1985). J. Chem. Soc. Perkin Trans. 2, pp. 847-852.

Gould, R. O. \& Walkinshaw, M. D. (1984). J. Am. Chem. Soc. 106, 7840-7842.

Groom, C. R., Bruno, I. J., Lightfoot, M. P. \& Ward, S. C. (2016). Acta Cryst. B72, 171-179.

Krishnan, P., Gayathri, K., Sivakumar, N., Chakkaravarthi, G. \& Anbalagan, G. (2013). Acta Cryst. E69, o659.

Krishnan, P., Gayathri, K., Sivakumar, N., Gunasekaran, B. \& Anbalagan, G. (2013). Acta Cryst. E69, o870.

Latham, K., Downs, J. E., Rix, C. J. \& White, J. M. (2011). J. Mol. Struct. 987, 74-85.

O'Neil, M. J. (2001). Editor. The Merck Index, 13th ed., p. 243. Whitehouse Station, NJ: Merck and Co., Inc.

Oshikawa, T., Pochamroen, S., Takai, N., Ida, N., Takemoto, T. \& Yamashita, M. (2002). Heterocycl. Commun. 8, 271-274.
Peerdeman, A. F. (1956). Acta Cryst. 9, 824.

Rigaku OD (2015). CrysAlis PRO. Rigaku Oxford Diffraction Ltd, Yarnton, England.

Sheldrick, G. M. (2008). Acta Cryst. A64, 112-122.

Smith, G. \& Wermuth, U. D. (2010). Acta Cryst. E66, o1893-o1894.

Smith, G. \& Wermuth, U. D. (2011). Acta Cryst. C67, o334-o336.

Smith, G. \& Wermuth, U. D. (2014). Acta Cryst. C70, 738-741.

Smith, G., Wermuth, U. D., Healy, P. C. \& White, J. M. (2006a). Acta Cryst. C62, o203-0207.

Smith, G., Wermuth, U. D., Healy, P. C. \& White, J. M. (2006b). Aust. J. Chem. 59, 321-328.

Smith, G., Wermuth, U. D., Healy, P. C., Young, D. J. \& White, J. M. (2005). Acta Cryst. E61, o2646-02648.

Smith, G., Wermuth, U. D. \& White, J. M. (2005). Acta Cryst. C61, o621-0624.

Smith, G., Wermuth, U. D. \& White, J. M. (2006). Acta Cryst. C62, o353-0357.

Smith, G., Wermuth, U. D. \& White, J. M. (2007). Acta Cryst. C63, o489-0492.

Smith, G., Wermuth, U. D. \& Williams, M. L. (2012). J. Chem. Crystallogr. 42, 555-559.

Smith, G., Wermuth, U. D. \& Young, D. J. (2010). J. Chem. Crystallogr. 40, 520-525.

Smith, G., Wermuth, U. D., Young, D. J. \& Healy, P. C. (2005). Acta Cryst. E61, o2008-o2011.

Smith, G., Wermuth, U. D., Young, D. J. \& White, J. M. (2006). Acta Cryst. E62, o1553-o1555.

Spek, A. L. (2009). Acta Cryst. D65, 148-155.

Steverding, D. (2010). Parasites Vectors, 3, 15.

Thomas, H. W. (1905). Proc. Roy. Soc. B: Biol. Sci. 76, 589-591.

Wilen, S. H. (1972). Tables of Resolving Agents and Optical Resolutions, edited by E. N. Eliel, pp. 68-71. London: University of Notre Dame. 


\section{supporting information}

Acta Cryst. (2016). E72, 751-755 [doi:10.1107/S2056989016006691]

\section{Crystal structure and hydrogen bonding in the water-stabilized proton-transfer}

\section{salt brucinium 4-aminophenylarsonate tetrahydrate}

\section{Graham Smith and Urs D. Wermuth}

Computing details

Data collection: CrysAlis PRO (Rigaku OD, 2015); cell refinement: CrysAlis PRO (Rigaku OD, 2015); data reduction:

CrysAlis PRO (Rigaku OD, 2015); program(s) used to solve structure: SIR92 (Altomare et al., 1993); program(s) used to refine structure: SHELXL97 (Sheldrick, 2008) within WinGX (Farrugia, 2012); molecular graphics: PLATON (Spek, 2009); software used to prepare material for publication: PLATON (Spek, 2009).

2,3-Dimethoxy-10-oxostrychnidinium 4-aminophenylarsonate tetrahydrate

Crystal data

$\left(\mathrm{C}_{23} \mathrm{H}_{27} \mathrm{~N}_{2} \mathrm{O}_{4}\right)\left[\mathrm{As}\left(\mathrm{C}_{6} \mathrm{H}_{7} \mathrm{~N}\right) \mathrm{O}_{2}(\mathrm{OH})\right] \cdot 4 \mathrm{H}_{2} \mathrm{O}$

$M_{r}=683.58$

Orthorhombic, $P 2_{1} 2_{1} 2_{1}$

Hall symbol: P 2ac 2ab

$a=7.6553(3) \AA$

$b=12.3238(5) \AA$

$c=31.960(2) \AA$

$V=3015.2(3) \AA^{3}$

$Z=4$

\section{Data collection}

Oxford Diffraction Gemini-S CCD-detector diffractometer

Radiation source: Enhance (Mo) X-ray source

Graphite monochromator

Detector resolution: 16.077 pixels $\mathrm{mm}^{-1}$

$\omega$ scans

Absorption correction: multi-scan

(CrysAlis PRO; Rigaku OD, 2015)

$T_{\min }=0.811, T_{\max }=0.980$

\section{Refinement}

Refinement on $F^{2}$

Least-squares matrix: full

$R\left[F^{2}>2 \sigma\left(F^{2}\right)\right]=0.048$

$w R\left(F^{2}\right)=0.096$

$S=1.05$

6980 reflections

433 parameters

14 restraints
$F(000)=1432$

$D_{\mathrm{x}}=1.506 \mathrm{Mg} \mathrm{m}^{-3}$

Mo $K \alpha$ radiation, $\lambda=0.71073 \AA$

Cell parameters from 2822 reflections

$\theta=3.4-27.9^{\circ}$

$\mu=1.19 \mathrm{~mm}^{-1}$

$T=200 \mathrm{~K}$

Plate, colourless

$0.36 \times 0.34 \times 0.10 \mathrm{~mm}$

11983 measured reflections

6980 independent reflections

5901 reflections with $I>2 \sigma(I)$

$R_{\text {int }}=0.032$

$\theta_{\max }=29.5^{\circ}, \theta_{\min }=3.1^{\circ}$

$h=-10 \rightarrow 6$

$k=-16 \rightarrow 16$

$l=-43 \rightarrow 25$

Primary atom site location: structure-invariant direct methods

Secondary atom site location: difference Fourier map

Hydrogen site location: inferred from neighbouring sites

$\mathrm{H}$ atoms treated by a mixture of independent and constrained refinement 
$w=1 /\left[\sigma^{2}\left(F_{\mathrm{o}}^{2}\right)+(0.0414 P)^{2}+0.2011 P\right]$

where $P=\left(F_{\mathrm{o}}^{2}+2 F_{\mathrm{c}}^{2}\right) / 3$

$(\Delta / \sigma)_{\max }=0.001$

$\Delta \rho_{\max }=0.55$ e $\AA^{-3}$
$\Delta \rho_{\min }=-0.46$ e $\AA^{-3}$

Absolute structure: Flack (1983), 3672 Friedel pairs

Absolute structure parameter: -0.005 (9)

\section{Special details}

Geometry. Bond distances, angles etc. have been calculated using the rounded fractional coordinates. All su's are estimated from the variances of the (full) variance-covariance matrix. The cell esds are taken into account in the estimation of distances, angles and torsion angles

Refinement. Refinement of $\mathrm{F}^{2}$ against ALL reflections. The weighted R-factor $\mathrm{wR}$ and goodness of fit $\mathrm{S}$ are based on $\mathrm{F}^{2}$, conventional R-factors $R$ are based on $F$, with $F$ set to zero for negative $\mathrm{F}^{2}$. The threshold expression of $\mathrm{F}^{2}>2 \operatorname{sigma}\left(\mathrm{F}^{2}\right)$ is used only for calculating R-factors(gt) etc. and is not relevant to the choice of reflections for refinement. R-factors based on $\mathrm{F}^{2}$ are statistically about twice as large as those based on F, and R- factors based on ALL data will be even larger.

Fractional atomic coordinates and isotropic or equivalent isotropic displacement parameters $\left(\AA^{2}\right)$

\begin{tabular}{|c|c|c|c|c|}
\hline & $x$ & $y$ & $z$ & $U_{\text {iso }} * / U_{\text {eq }}$ \\
\hline $\mathrm{O} 2$ & $0.2664(3)$ & $0.56076(19)$ & $0.24172(7)$ & $0.0288(8)$ \\
\hline $\mathrm{O} 3$ & $0.2324(3)$ & $0.44912(19)$ & $0.17363(7)$ & $0.0251(7)$ \\
\hline $\mathrm{O} 24$ & 0.2010 & $-0.13571(17)$ & $0.32202(7)$ & $0.0224(7)$ \\
\hline $\mathrm{O} 25$ & $0.2193(4)$ & $0.0496(2)$ & $0.19539(7)$ & $0.0336(9)$ \\
\hline N9 & $0.1591(3)$ & $0.1193(2)$ & $0.25929(8)$ & $0.0192(7)$ \\
\hline N19 & $0.1326(4)$ & $0.2044(2)$ & $0.39973(8)$ & $0.0220(8)$ \\
\hline $\mathrm{C} 1$ & $0.2107(4)$ & $0.4025(3)$ & $0.28549(10)$ & $0.0220(10)$ \\
\hline $\mathrm{C} 2$ & $0.2316(4)$ & 0.4525 & $0.24709(10)$ & $0.0206(10)$ \\
\hline $\mathrm{C} 3$ & $0.2176(4)$ & $0.3914(3)$ & $0.21006(10)$ & $0.0200(9)$ \\
\hline $\mathrm{C} 4$ & $0.1912(4)$ & $0.2806(2)$ & $0.21125(9)$ & $0.0192(9)$ \\
\hline $\mathrm{C} 5$ & $0.1762(5)$ & $0.2319(2)$ & $0.25023(10)$ & $0.0186(9)$ \\
\hline C6 & $0.1822(5)$ & $0.2909(3)$ & $0.28690(9)$ & $0.0200(9)$ \\
\hline $\mathrm{C} 7$ & $0.1402(4)$ & $0.2196(3)$ & $0.32382(10)$ & $0.0194(9)$ \\
\hline $\mathrm{C} 8$ & $0.1639(4)$ & $0.1035(3)$ & $0.30544(9)$ & $0.0178(9)$ \\
\hline $\mathrm{C} 10$ & $0.2084(5)$ & $0.0380(3)$ & $0.23349(10)$ & $0.0224(10)$ \\
\hline $\mathrm{C} 11$ & $0.2482(5)$ & $-0.0701(3)$ & $0.25362(11)$ & $0.0244(11)$ \\
\hline $\mathrm{C} 12$ & $0.3195(5)$ & $-0.0703(3)$ & $0.29876(10)$ & $0.0216(10)$ \\
\hline $\mathrm{C} 13$ & $0.3369(4)$ & $0.0468(3)$ & $0.31477(9)$ & $0.0173(9)$ \\
\hline $\mathrm{C} 14$ & $0.3946(4)$ & $0.0634(3)$ & $0.36027(10)$ & $0.0208(10)$ \\
\hline C15 & $0.4243(4)$ & $0.1858(3)$ & $0.36540(11)$ & $0.0217(10)$ \\
\hline C16 & $0.2486(5)$ & $0.2415(3)$ & $0.36351(10)$ & $0.0215(10)$ \\
\hline $\mathrm{C} 17$ & $-0.0479(4)$ & 0.2361 & $0.33974(11)$ & $0.0236(11)$ \\
\hline C18 & -0.0461 (4) & 0.1812 & $0.38190(10)$ & $0.0236(10)$ \\
\hline $\mathrm{C} 20$ & $0.2066(5)$ & 0.1088 (3) & $0.42293(9)$ & $0.0234(10)$ \\
\hline $\mathrm{C} 21$ & $0.2646(4)$ & $0.0242(3)$ & $0.39246(10)$ & $0.0221(10)$ \\
\hline $\mathrm{C} 22$ & $0.2076(5)$ & -0.0761 & $0.39424(10)$ & $0.0235(10)$ \\
\hline $\mathrm{C} 23$ & $0.2581(5)$ & -0.1618 & $0.36323(11)$ & $0.0269(11)$ \\
\hline $\mathrm{C} 25$ & $0.2845(6)$ & $0.6248(3)$ & $0.27850(12)$ & $0.0400(14)$ \\
\hline $\mathrm{C} 26$ & $0.2222(4)$ & $0.3880(3)$ & $0.13581(10)$ & $0.0263(10)$ \\
\hline As1A & $0.18853(4)$ & $0.38087(2)$ & 0.50015 (1) & $0.0194(1)$ \\
\hline O11A & $0.0706(3)$ & $0.2967(2)$ & $0.52906(7)$ & $0.0288(8)$ \\
\hline O12A & $0.1351(3)$ & 0.37219 (19) & $0.44956(7)$ & $0.0256(7)$ \\
\hline
\end{tabular}




\begin{tabular}{|c|c|c|c|c|}
\hline O13A & $0.4046(3)$ & $0.3544(2)$ & $0.50798(9)$ & $0.0361(9)$ \\
\hline $\mathrm{N} 4 \mathrm{~A}$ & $0.1284(6)$ & $0.8469(3)$ & $0.55939(14)$ & $0.0526(15)$ \\
\hline $\mathrm{C} 1 \mathrm{~A}$ & $0.1723(5)$ & $0.5265(2)$ & $0.51885(9)$ & $0.0213(9)$ \\
\hline $\mathrm{C} 2 \mathrm{~A}$ & $0.0081(5)$ & $0.5733(3)$ & $0.52485(11)$ & $0.0277(11)$ \\
\hline $\mathrm{C} 3 \mathrm{~A}$ & $-0.0043(6)$ & $0.6792(3)$ & $0.53827(11)$ & $0.0320(12)$ \\
\hline $\mathrm{C} 4 \mathrm{~A}$ & $0.1423(6)$ & $0.7411(3)$ & $0.54628(12)$ & $0.0314(13)$ \\
\hline $\mathrm{C} 5 \mathrm{~A}$ & $0.3047(6)$ & $0.6939(3)$ & $0.53962(11)$ & $0.0324(11)$ \\
\hline C6A & $0.3193(5)$ & $0.5885(3)$ & $0.52554(10)$ & $0.0271(10)$ \\
\hline $\mathrm{O} 1 \mathrm{~W}$ & $0.4311(4)$ & $-0.0600(3)$ & $0.13578(10)$ & $0.0461(11)$ \\
\hline $\mathrm{O} 2 \mathrm{~W}$ & $-0.2441(4)$ & $0.3881(3)$ & 0.43528 & $0.0521(11)$ \\
\hline $\mathrm{O} 3 \mathrm{~W}$ & $0.4514(4)$ & $0.8770(3)$ & $0.61869(11)$ & $0.0587(12)$ \\
\hline $\mathrm{O} 4 \mathrm{~W}$ & $0.2795(4)$ & $0.5374(3)$ & $0.40023(10)$ & $0.0511(11)$ \\
\hline H1 & 0.21570 & 0.44380 & 0.31060 & $0.0260^{*}$ \\
\hline $\mathrm{H} 4$ & 0.18370 & 0.23920 & 0.18630 & $0.0230 *$ \\
\hline $\mathrm{H} 8$ & 0.06440 & 0.05630 & 0.31430 & $0.0210^{*}$ \\
\hline H12 & 0.43720 & -0.10550 & 0.29900 & $0.0260^{*}$ \\
\hline H13 & 0.42710 & 0.08270 & 0.29690 & $0.0210^{*}$ \\
\hline H14 & 0.50800 & 0.02480 & 0.36480 & $0.0250 *$ \\
\hline H16 & 0.26740 & 0.32150 & 0.36610 & $0.0260 *$ \\
\hline H19 & $0.122(6)$ & $0.258(3)$ & $0.4190(11)$ & $0.0620 *$ \\
\hline $\mathrm{H} 22$ & 0.13050 & -0.09540 & 0.41630 & $0.0280^{*}$ \\
\hline H111 & 0.33410 & -0.10810 & 0.23570 & $0.0290^{*}$ \\
\hline H112 & 0.13960 & -0.11370 & 0.25330 & $0.0290^{*}$ \\
\hline H151 & 0.48150 & 0.20080 & 0.39260 & $0.0260 *$ \\
\hline H152 & 0.50090 & 0.21300 & 0.34270 & $0.0260 *$ \\
\hline H171 & -0.07610 & 0.31420 & 0.34240 & $0.0280^{*}$ \\
\hline H172 & -0.13360 & 0.20150 & 0.32080 & $0.0280^{*}$ \\
\hline H181 & -0.06470 & 0.10210 & 0.37890 & $0.0280^{*}$ \\
\hline H182 & -0.13850 & 0.21120 & 0.40020 & $0.0280^{*}$ \\
\hline $\mathrm{H} 201$ & 0.30700 & 0.13230 & 0.44020 & $0.0280^{*}$ \\
\hline $\mathrm{H} 202$ & 0.11670 & 0.07810 & 0.44180 & $0.0280^{*}$ \\
\hline $\mathrm{H} 231$ & 0.38680 & -0.17010 & 0.36320 & $0.0320^{*}$ \\
\hline $\mathrm{H} 232$ & 0.20630 & -0.23190 & 0.37180 & $0.0320^{*}$ \\
\hline $\mathrm{H} 251$ & 0.30870 & 0.70020 & 0.27070 & $0.0600^{*}$ \\
\hline $\mathrm{H} 252$ & 0.38120 & 0.59660 & 0.29540 & $0.0600^{*}$ \\
\hline $\mathrm{H} 253$ & 0.17600 & 0.62160 & 0.29470 & $0.0600^{*}$ \\
\hline $\mathrm{H} 261$ & 0.23380 & 0.43690 & 0.11180 & $0.0390^{*}$ \\
\hline $\mathrm{H} 262$ & 0.10930 & 0.35070 & 0.13440 & $0.0390 *$ \\
\hline $\mathrm{H} 263$ & 0.31660 & 0.33430 & 0.13520 & $0.0390 *$ \\
\hline $\mathrm{H} 2 \mathrm{~A}$ & -0.09470 & 0.53230 & 0.51970 & $0.0330 *$ \\
\hline $\mathrm{H} 3 \mathrm{~A}$ & -0.11650 & 0.71040 & 0.54210 & $0.0390 *$ \\
\hline $\mathrm{H} 5 \mathrm{~A}$ & 0.40740 & 0.73490 & 0.54490 & $0.0390 *$ \\
\hline H6A & 0.43160 & 0.55850 & 0.52040 & $0.0330^{*}$ \\
\hline H13A & $0.445(6)$ & 0.298 & $0.4931(13)$ & $0.0770^{*}$ \\
\hline $\mathrm{H} 41 \mathrm{~A}$ & $0.022(3)$ & $0.876(4)$ & $0.5617(15)$ & $0.0620^{*}$ \\
\hline $\mathrm{H} 42 \mathrm{~A}$ & $0.227(3)$ & $0.861(4)$ & $0.5735(13)$ & $0.0620^{*}$ \\
\hline H11W & $0.360(5)$ & -0.029 & $0.1548(10)$ & $0.0770^{*}$ \\
\hline $\mathrm{H} 12 \mathrm{~W}$ & $0.358(5)$ & $-0.071(4)$ & $0.1141(10)$ & $0.0770^{*}$ \\
\hline
\end{tabular}




\begin{tabular}{|c|c|c|c|c|}
\hline H21W & $-0.134(3)$ & $0.406(4)$ & $0.4425(16)$ & $0.0770 *$ \\
\hline H22W & $-0.273(7)$ & $0.328(2)$ & $0.4492(14)$ & $0.0770 *$ \\
\hline H31W & $0.406(6)$ & $0.917(3)$ & $0.6400(11)$ & $0.0770 *$ \\
\hline H32W & $0.548(4)$ & $0.917(3)$ & $0.6129(15)$ & $0.0770 *$ \\
\hline H41W & $0.378(4)$ & $0.512(4)$ & $0.3885(15)$ & $0.0770^{*}$ \\
\hline H42W & $0.242(7)$ & $0.483(3)$ & $0.4163(13)$ & $0.0770 *$ \\
\hline
\end{tabular}

Atomic displacement parameters $\left(\AA^{2}\right)$

\begin{tabular}{|c|c|c|c|c|c|c|}
\hline & $U^{11}$ & $U^{22}$ & $U^{33}$ & $U^{12}$ & $U^{13}$ & $U^{23}$ \\
\hline $\mathrm{O} 2$ & $0.0412(17)$ & 0.0165 (12) & 0.0288 & $-0.0036(11)$ & 0.0025 (12) & $-0.0002(10)$ \\
\hline $\mathrm{O} 3$ & $0.0292(13)$ & 0.0255 (13) & 0.0207 (12) & $-0.0021(11)$ & $0.0027(10)$ & $0.0008(10)$ \\
\hline $\mathrm{O} 24$ & $0.0244(12)$ & 0.0185 & $0.0243(11)$ & $-0.0011(11)$ & $-0.0001(11)$ & $0.0000(9)$ \\
\hline $\mathrm{O} 25$ & $0.0533(19)$ & $0.0281(14)$ & $0.0195(12)$ & $0.0026(14)$ & 0.0064 (13) & $-0.0055(10)$ \\
\hline N9 & $0.0226(14)$ & $0.0182(12)$ & $0.0168(12)$ & $0.0001(13)$ & $-0.0003(11)$ & $-0.0035(11)$ \\
\hline N19 & $0.0261(15)$ & $0.0230(15)$ & 0.0169 (14) & $-0.0009(12)$ & $0.0011(12)$ & $-0.0052(12)$ \\
\hline $\mathrm{C} 1$ & $0.0251(19)$ & 0.0207 (17) & $0.0203(16)$ & $0.0005(14)$ & $-0.0011(15)$ & $-0.0087(13)$ \\
\hline $\mathrm{C} 2$ & $0.0181(18)$ & $0.0173(16)$ & $0.0263(18)$ & $0.0022(13)$ & $0.0011(15)$ & $0.0004(14)$ \\
\hline C3 & $0.0169(17)$ & $0.0247(17)$ & $0.0183(15)$ & $-0.0004(15)$ & $0.0021(13)$ & $0.0018(14)$ \\
\hline $\mathrm{C} 4$ & $0.0200(16)$ & $0.0229(16)$ & $0.0148(14)$ & $-0.0001(15)$ & $0.0007(15)$ & $-0.0046(12)$ \\
\hline $\mathrm{C} 5$ & $0.0196(17)$ & $0.0181(15)$ & $0.0181(15)$ & $0.0014(14)$ & $-0.0008(15)$ & $-0.0012(12)$ \\
\hline C6 & $0.0196(16)$ & $0.0213(15)$ & $0.0192(15)$ & $0.0022(15)$ & $0.0023(15)$ & $0.0000(13)$ \\
\hline $\mathrm{C} 7$ & $0.0228(17)$ & $0.0176(16)$ & $0.0177(16)$ & $0.0017(13)$ & 0.0007 (14) & $-0.0031(13)$ \\
\hline $\mathrm{C} 8$ & $0.0193(16)$ & $0.0188(16)$ & $0.0153(14)$ & $-0.0002(14)$ & $0.0004(13)$ & $-0.0039(12)$ \\
\hline $\mathrm{C} 10$ & $0.0214(18)$ & $0.0235(17)$ & $0.0224(17)$ & $-0.0025(16)$ & $0.0005(16)$ & $-0.0059(14)$ \\
\hline C11 & $0.030(2)$ & $0.0184(17)$ & $0.0248(18)$ & $0.0021(14)$ & $-0.0014(16)$ & $-0.0066(14)$ \\
\hline $\mathrm{C} 12$ & $0.0204(17)$ & $0.0190(16)$ & $0.0255(17)$ & $0.0035(16)$ & $0.0024(16)$ & $-0.0047(13)$ \\
\hline C13 & $0.0137(16)$ & $0.0175(15)$ & $0.0208(16)$ & $-0.0005(13)$ & $0.0031(13)$ & $-0.0038(12)$ \\
\hline $\mathrm{C} 14$ & $0.0164(17)$ & 0.0248 (18) & $0.0211(17)$ & $0.0020(14)$ & $-0.0029(14)$ & $-0.0028(14)$ \\
\hline $\mathrm{C} 15$ & $0.0210(18)$ & $0.0250(18)$ & $0.0192(17)$ & $-0.0039(15)$ & $-0.0021(15)$ & $-0.0058(15)$ \\
\hline $\mathrm{C} 16$ & $0.0291(18)$ & $0.0173(16)$ & $0.0182(16)$ & $-0.0043(13)$ & $0.0024(15)$ & $-0.0043(13)$ \\
\hline $\mathrm{C} 17$ & 0.0249 (19) & 0.0242 (19) & $0.0216(17)$ & $0.0053(15)$ & $0.0030(15)$ & $-0.0054(14)$ \\
\hline C18 & $0.0186(17)$ & 0.0283 (19) & $0.0239(18)$ & $0.0018(15)$ & $0.0061(15)$ & $-0.0032(15)$ \\
\hline $\mathrm{C} 20$ & $0.0289(18)$ & $0.0239(17)$ & $0.0175(15)$ & $-0.0004(17)$ & $-0.0029(15)$ & $0.0001(14)$ \\
\hline $\mathrm{C} 21$ & $0.0204(17)$ & 0.0257 (18) & $0.0201(16)$ & $0.0025(14)$ & $-0.0065(14)$ & $0.0003(14)$ \\
\hline $\mathrm{C} 22$ & $0.0229(18)$ & $0.0272(17)$ & $0.0205(16)$ & $0.0019(15)$ & $-0.0026(16)$ & $0.0032(13)$ \\
\hline $\mathrm{C} 23$ & 0.0284 (19) & $0.0210(17)$ & 0.0314 (19) & $-0.0009(14)$ & $-0.0039(16)$ & $0.0031(15)$ \\
\hline $\mathrm{C} 25$ & $0.062(3)$ & 0.0220 (19) & $0.036(2)$ & $-0.004(2)$ & $-0.002(2)$ & $-0.0032(17)$ \\
\hline $\mathrm{C} 26$ & $0.0268(19)$ & 0.0317 (19) & $0.0204(15)$ & $0.0005(17)$ & $-0.0019(14)$ & $0.0030(16)$ \\
\hline As1A & $0.0219(2)$ & 0.0175 (1) & 0.0187 (1) & -0.0005 & $0.0006(2)$ & $-0.0038(2)$ \\
\hline O11A & $0.0363(15)$ & $0.0273(13)$ & $0.0229(12)$ & $-0.0063(12)$ & $0.0047(11)$ & $-0.0041(11)$ \\
\hline $\mathrm{O} 12 \mathrm{~A}$ & $0.0368(14)$ & $0.0204(12)$ & $0.0197(11)$ & $0.0008(11)$ & $0.0016(10)$ & $-0.0054(10)$ \\
\hline O13A & $0.0239(12)$ & 0.0339 (14) & 0.0505 (19) & 0.0038 (11) & $-0.0055(13)$ & $-0.0178(13)$ \\
\hline N4A & $0.060(3)$ & 0.0279 (19) & $0.070(3)$ & $0.0095(18)$ & $-0.011(2)$ & $-0.0174(18)$ \\
\hline $\mathrm{C} 1 \mathrm{~A}$ & $0.0328(19)$ & $0.0171(15)$ & $0.0139(15)$ & $-0.0015(15)$ & $0.0001(16)$ & $-0.0007(12)$ \\
\hline $\mathrm{C} 2 \mathrm{~A}$ & $0.0270(19)$ & 0.0250 (19) & $0.031(2)$ & $0.0009(15)$ & 0.0045 (17) & $-0.0009(16)$ \\
\hline $\mathrm{C} 3 \mathrm{~A}$ & $0.042(2)$ & $0.026(2)$ & $0.028(2)$ & $0.0090(17)$ & $0.0073(18)$ & $0.0008(16)$ \\
\hline $\mathrm{C} 4 \mathrm{~A}$ & $0.048(3)$ & $0.0208(18)$ & $0.0254(18)$ & $0.0027(17)$ & $-0.0018(18)$ & $0.0023(15)$ \\
\hline
\end{tabular}




\begin{tabular}{lllllll} 
C5A & $0.043(2)$ & $0.0239(18)$ & $0.0303(19)$ & $-0.0078(19)$ & $-0.0059(19)$ & $-0.0012(15)$ \\
C6A & $0.0313(19)$ & $0.0277(18)$ & $0.0224(16)$ & $-0.0030(17)$ & $-0.0018(18)$ & $-0.0022(14)$ \\
O1W & $0.0439(18)$ & $0.0500(19)$ & $0.0445(18)$ & $0.0115(16)$ & $0.0045(15)$ & $-0.0085(16)$ \\
O2W & $0.0453(17)$ & $0.053(2)$ & $0.058(2)$ & $0.0021(17)$ & $0.0002(16)$ & $0.0257(18)$ \\
O3W & $0.059(2)$ & $0.059(2)$ & $0.058(2)$ & $-0.0071(19)$ & $0.0088(17)$ & $-0.0055(18)$ \\
O4W & $0.050(2)$ & $0.0452(19)$ & $0.058(2)$ & $0.0023(16)$ & $0.0108(17)$ & $0.0184(16)$ \\
\hline
\end{tabular}

Geometric parameters $\left(\AA,{ }^{\circ}\right)$

\begin{tabular}{|c|c|c|c|}
\hline $\mathrm{As} 1 \mathrm{~A}-\mathrm{O} 12 \mathrm{~A}$ & $1.671(2)$ & $\mathrm{C} 13-\mathrm{C} 14$ & $1.534(4)$ \\
\hline As $1 \mathrm{~A}-\mathrm{O} 13 \mathrm{~A}$ & $1.704(2)$ & $\mathrm{C} 14-\mathrm{C} 21$ & $1.511(5)$ \\
\hline $\mathrm{As} 1 \mathrm{~A}-\mathrm{C} 1 \mathrm{~A}$ & $1.896(3)$ & $\mathrm{C} 14-\mathrm{C} 15$ & $1.534(5)$ \\
\hline As $1 \mathrm{~A}-\mathrm{O} 11 \mathrm{~A}$ & $1.657(2)$ & $\mathrm{C} 15-\mathrm{C} 16$ & $1.511(5)$ \\
\hline $\mathrm{O} 2-\mathrm{C} 2$ & $1.371(4)$ & $\mathrm{C} 17-\mathrm{C} 18$ & $1.508(5)$ \\
\hline $\mathrm{O} 2-\mathrm{C} 25$ & $1.423(4)$ & $\mathrm{C} 20-\mathrm{C} 21$ & $1.494(5)$ \\
\hline $\mathrm{O} 3-\mathrm{C} 26$ & $1.426(4)$ & $\mathrm{C} 21-\mathrm{C} 22$ & $1.312(5)$ \\
\hline $\mathrm{O} 3-\mathrm{C} 3$ & $1.369(4)$ & $\mathrm{C} 22-\mathrm{C} 23$ & $1.499(5)$ \\
\hline $\mathrm{O} 24-\mathrm{C} 23$ & $1.425(4)$ & $\mathrm{C} 1-\mathrm{H} 1$ & 0.9500 \\
\hline $\mathrm{O} 24-\mathrm{C} 12$ & $1.423(4)$ & $\mathrm{C} 4-\mathrm{H} 4$ & 0.9500 \\
\hline $\mathrm{O} 25-\mathrm{C} 10$ & $1.229(4)$ & $\mathrm{C} 8-\mathrm{H} 8$ & 1.0000 \\
\hline $\mathrm{O} 13 \mathrm{~A}-\mathrm{H} 13 \mathrm{~A}$ & $0.90(4)$ & C11-H111 & 0.9900 \\
\hline $\mathrm{O} 1 \mathrm{~W}-\mathrm{H} 12 \mathrm{~W}$ & $0.90(3)$ & $\mathrm{C} 11-\mathrm{H} 112$ & 0.9900 \\
\hline $\mathrm{O} 1 \mathrm{~W}-\mathrm{H} 11 \mathrm{~W}$ & $0.90(4)$ & $\mathrm{C} 12-\mathrm{H} 12$ & 1.0000 \\
\hline $\mathrm{O} 2 \mathrm{~W}-\mathrm{H} 22 \mathrm{~W}$ & $0.89(3)$ & $\mathrm{C} 13-\mathrm{H} 13$ & 1.0000 \\
\hline $\mathrm{O} 2 \mathrm{~W}-\mathrm{H} 21 \mathrm{~W}$ & $0.90(3)$ & $\mathrm{C} 14-\mathrm{H} 14$ & 1.0000 \\
\hline $\mathrm{O} 3 \mathrm{~W}-\mathrm{H} 32 \mathrm{~W}$ & $0.91(3)$ & C15-H152 & 0.9900 \\
\hline $\mathrm{O} 3 \mathrm{~W}-\mathrm{H} 31 \mathrm{~W}$ & $0.91(4)$ & $\mathrm{C} 15-\mathrm{H} 151$ & 0.9900 \\
\hline $\mathrm{N} 9-\mathrm{C} 5$ & $1.424(4)$ & $\mathrm{C} 16-\mathrm{H} 16$ & 1.0000 \\
\hline $\mathrm{N} 9-\mathrm{C} 10$ & $1.351(4)$ & $\mathrm{C} 17-\mathrm{H} 171$ & 0.9900 \\
\hline $\mathrm{N} 9-\mathrm{C} 8$ & $1.488(4)$ & C17-H172 & 0.9900 \\
\hline $\mathrm{N} 19-\mathrm{C} 16$ & $1.529(4)$ & C18-H182 & 0.9900 \\
\hline N19-C18 & $1.509(4)$ & C18-H181 & 0.9900 \\
\hline N19-C20 & $1.503(4)$ & $\mathrm{C} 20-\mathrm{H} 202$ & 0.9900 \\
\hline $\mathrm{O} 4 \mathrm{~W}-\mathrm{H} 42 \mathrm{~W}$ & $0.89(4)$ & $\mathrm{C} 20-\mathrm{H} 201$ & 0.9900 \\
\hline $\mathrm{O} 4 \mathrm{~W}-\mathrm{H} 41 \mathrm{~W}$ & $0.90(4)$ & $\mathrm{C} 22-\mathrm{H} 22$ & 0.9500 \\
\hline N19-H19 & $0.91(4)$ & $\mathrm{C} 23-\mathrm{H} 232$ & 0.9900 \\
\hline $\mathrm{N} 4 \mathrm{~A}-\mathrm{C} 4 \mathrm{~A}$ & $1.374(5)$ & $\mathrm{C} 23-\mathrm{H} 231$ & 0.9900 \\
\hline $\mathrm{N} 4 \mathrm{~A}-\mathrm{H} 41 \mathrm{~A}$ & $0.89(3)$ & $\mathrm{C} 25-\mathrm{H} 252$ & 0.9800 \\
\hline $\mathrm{N} 4 \mathrm{~A}-\mathrm{H} 42 \mathrm{~A}$ & $0.90(3)$ & $\mathrm{C} 25-\mathrm{H} 251$ & 0.9800 \\
\hline $\mathrm{C} 1-\mathrm{C} 2$ & $1.383(5)$ & $\mathrm{C} 25-\mathrm{H} 253$ & 0.9800 \\
\hline $\mathrm{C} 1-\mathrm{C} 6$ & $1.393(5)$ & $\mathrm{C} 26-\mathrm{H} 262$ & 0.9800 \\
\hline $\mathrm{C} 2-\mathrm{C} 3$ & $1.407(5)$ & $\mathrm{C} 26-\mathrm{H} 263$ & 0.9800 \\
\hline $\mathrm{C} 3-\mathrm{C} 4$ & $1.381(4)$ & $\mathrm{C} 26-\mathrm{H} 261$ & 0.9800 \\
\hline $\mathrm{C} 4-\mathrm{C} 5$ & $1.388(4)$ & $\mathrm{C} 1 \mathrm{~A}-\mathrm{C} 6 \mathrm{~A}$ & $1.377(5)$ \\
\hline $\mathrm{C} 5-\mathrm{C} 6$ & $1.380(4)$ & $\mathrm{C} 1 \mathrm{~A}-\mathrm{C} 2 \mathrm{~A}$ & $1.396(5)$ \\
\hline $\mathrm{C} 6-\mathrm{C} 7$ & $1.506(5)$ & $\mathrm{C} 2 \mathrm{~A}-\mathrm{C} 3 \mathrm{~A}$ & $1.377(5)$ \\
\hline $\mathrm{C} 7-\mathrm{C} 17$ & $1.541(4)$ & $\mathrm{C} 3 \mathrm{~A}-\mathrm{C} 4 \mathrm{~A}$ & $1.381(6)$ \\
\hline $\mathrm{C} 7-\mathrm{C} 16$ & $1.540(5)$ & $\mathrm{C} 4 \mathrm{~A}-\mathrm{C} 5 \mathrm{~A}$ & $1.389(6)$ \\
\hline
\end{tabular}




\begin{tabular}{|c|c|c|c|}
\hline $\mathrm{C} 7-\mathrm{C} 8$ & $1.557(5)$ & $\mathrm{C} 5 \mathrm{~A}-\mathrm{C} 6 \mathrm{~A}$ & $1.379(5)$ \\
\hline $\mathrm{C} 8-\mathrm{C} 13$ & $1.527(5)$ & $\mathrm{C} 2 \mathrm{~A}-\mathrm{H} 2 \mathrm{~A}$ & 0.9500 \\
\hline $\mathrm{C} 10-\mathrm{C} 11$ & $1.511(5)$ & $\mathrm{C} 3 \mathrm{~A}-\mathrm{H} 3 \mathrm{~A}$ & 0.9500 \\
\hline $\mathrm{C} 11-\mathrm{C} 12$ & $1.543(5)$ & $\mathrm{C} 5 \mathrm{~A}-\mathrm{H} 5 \mathrm{~A}$ & 0.9500 \\
\hline $\mathrm{C} 12-\mathrm{C} 13$ & $1.537(5)$ & $\mathrm{C} 6 \mathrm{~A}-\mathrm{H} 6 \mathrm{~A}$ & 0.9500 \\
\hline $\mathrm{O} 12 \mathrm{~A}-\mathrm{As} 1 \mathrm{~A}-\mathrm{C} 1 \mathrm{~A}$ & $110.46(12)$ & $\mathrm{N} 9-\mathrm{C} 8-\mathrm{H} 8$ & 110.00 \\
\hline $\mathrm{O} 13 \mathrm{~A}-\mathrm{As} 1 \mathrm{~A}-\mathrm{C} 1 \mathrm{~A}$ & $101.45(14)$ & $\mathrm{C} 7-\mathrm{C} 8-\mathrm{H} 8$ & 110.00 \\
\hline $\mathrm{O} 12 \mathrm{~A}-\mathrm{As} 1 \mathrm{~A}-\mathrm{O} 13 \mathrm{~A}$ & $111.55(13)$ & $\mathrm{C} 13-\mathrm{C} 8-\mathrm{H} 8$ & 110.00 \\
\hline $\mathrm{O} 11 \mathrm{~A}-\mathrm{As} 1 \mathrm{~A}-\mathrm{C} 1 \mathrm{~A}$ & $112.41(13)$ & $\mathrm{C} 12-\mathrm{C} 11-\mathrm{H} 111$ & 108.00 \\
\hline $\mathrm{O} 11 \mathrm{~A}-\mathrm{As} 1 \mathrm{~A}-\mathrm{O} 12 \mathrm{~A}$ & $111.48(11)$ & $\mathrm{C} 10-\mathrm{C} 11-\mathrm{H} 111$ & 108.00 \\
\hline O11A-As1A-O13A & $109.09(12)$ & $\mathrm{C} 10-\mathrm{C} 11-\mathrm{H} 112$ & 108.00 \\
\hline $\mathrm{C} 2-\mathrm{O} 2-\mathrm{C} 25$ & $117.1(3)$ & $\mathrm{H} 111-\mathrm{C} 11-\mathrm{H} 112$ & 107.00 \\
\hline $\mathrm{C} 3-\mathrm{O} 3-\mathrm{C} 26$ & $116.2(3)$ & $\mathrm{C} 12-\mathrm{C} 11-\mathrm{H} 112$ & 108.00 \\
\hline $\mathrm{C} 12-\mathrm{O} 24-\mathrm{C} 23$ & $114.5(3)$ & $\mathrm{O} 24-\mathrm{C} 12-\mathrm{H} 12$ & 109.00 \\
\hline As1A-O13A-H13A & $114(3)$ & $\mathrm{C} 13-\mathrm{C} 12-\mathrm{H} 12$ & 109.00 \\
\hline $\mathrm{H} 11 \mathrm{~W}-\mathrm{O} 1 \mathrm{~W}-\mathrm{H} 12 \mathrm{~W}$ & $102(3)$ & $\mathrm{C} 11-\mathrm{C} 12-\mathrm{H} 12$ & 109.00 \\
\hline $\mathrm{H} 21 \mathrm{~W}-\mathrm{O} 2 \mathrm{~W}-\mathrm{H} 22 \mathrm{~W}$ & $108(5)$ & $\mathrm{C} 8-\mathrm{C} 13-\mathrm{H} 13$ & 107.00 \\
\hline $\mathrm{H} 31 \mathrm{~W}-\mathrm{O} 3 \mathrm{~W}-\mathrm{H} 32 \mathrm{~W}$ & $100(4)$ & $\mathrm{C} 12-\mathrm{C} 13-\mathrm{H} 13$ & 107.00 \\
\hline $\mathrm{C} 8-\mathrm{N} 9-\mathrm{C} 10$ & $120.1(3)$ & $\mathrm{C} 14-\mathrm{C} 13-\mathrm{H} 13$ & 106.00 \\
\hline $\mathrm{C} 5-\mathrm{N} 9-\mathrm{C} 10$ & $125.0(3)$ & $\mathrm{C} 15-\mathrm{C} 14-\mathrm{H} 14$ & 109.00 \\
\hline $\mathrm{C} 5-\mathrm{N} 9-\mathrm{C} 8$ & $109.1(2)$ & $\mathrm{C} 21-\mathrm{C} 14-\mathrm{H} 14$ & 109.00 \\
\hline $\mathrm{C} 16-\mathrm{N} 19-\mathrm{C} 18$ & $107.3(2)$ & $\mathrm{C} 13-\mathrm{C} 14-\mathrm{H} 14$ & 109.00 \\
\hline $\mathrm{C} 16-\mathrm{N} 19-\mathrm{C} 20$ & $112.9(3)$ & $\mathrm{H} 151-\mathrm{C} 15-\mathrm{H} 152$ & 109.00 \\
\hline $\mathrm{C} 18-\mathrm{N} 19-\mathrm{C} 20$ & $112.3(3)$ & $\mathrm{C} 16-\mathrm{C} 15-\mathrm{H} 151$ & 110.00 \\
\hline $\mathrm{H} 41 \mathrm{~W}-\mathrm{O} 4 \mathrm{~W}-\mathrm{H} 42 \mathrm{~W}$ & $104(4)$ & $\mathrm{C} 16-\mathrm{C} 15-\mathrm{H} 152$ & 110.00 \\
\hline $\mathrm{C} 20-\mathrm{N} 19-\mathrm{H} 19$ & $106(2)$ & $\mathrm{C} 14-\mathrm{C} 15-\mathrm{H} 151$ & 110.00 \\
\hline C18-N19-H19 & $108(3)$ & $\mathrm{C} 14-\mathrm{C} 15-\mathrm{H} 152$ & 110.00 \\
\hline C16-N19-H19 & $110(3)$ & $\mathrm{N} 19-\mathrm{C} 16-\mathrm{H} 16$ & 108.00 \\
\hline $\mathrm{H} 41 \mathrm{~A}-\mathrm{N} 4 \mathrm{~A}-\mathrm{H} 42 \mathrm{~A}$ & $130(4)$ & $\mathrm{C} 15-\mathrm{C} 16-\mathrm{H} 16$ & 108.00 \\
\hline $\mathrm{C} 4 \mathrm{~A}-\mathrm{N} 4 \mathrm{~A}-\mathrm{H} 42 \mathrm{~A}$ & $106(3)$ & $\mathrm{C} 7-\mathrm{C} 16-\mathrm{H} 16$ & 109.00 \\
\hline $\mathrm{C} 4 \mathrm{~A}-\mathrm{N} 4 \mathrm{~A}-\mathrm{H} 41 \mathrm{~A}$ & $119(3)$ & $\mathrm{C} 7-\mathrm{C} 17-\mathrm{H} 171$ & 111.00 \\
\hline $\mathrm{C} 2-\mathrm{C} 1-\mathrm{C} 6$ & $119.1(3)$ & $\mathrm{C} 7-\mathrm{C} 17-\mathrm{H} 172$ & 111.00 \\
\hline $\mathrm{O} 2-\mathrm{C} 2-\mathrm{C} 1$ & $124.6(3)$ & $\mathrm{C} 18-\mathrm{C} 17-\mathrm{H} 171$ & 111.00 \\
\hline $\mathrm{O} 2-\mathrm{C} 2-\mathrm{C} 3$ & $115.5(3)$ & $\mathrm{C} 18-\mathrm{C} 17-\mathrm{H} 172$ & 111.00 \\
\hline $\mathrm{C} 1-\mathrm{C} 2-\mathrm{C} 3$ & $120.0(3)$ & $\mathrm{H} 171-\mathrm{C} 17-\mathrm{H} 172$ & 109.00 \\
\hline $\mathrm{O} 3-\mathrm{C} 3-\mathrm{C} 2$ & $115.5(3)$ & $\mathrm{H} 181-\mathrm{C} 18-\mathrm{H} 182$ & 109.00 \\
\hline $\mathrm{O} 3-\mathrm{C} 3-\mathrm{C} 4$ & $123.3(3)$ & $\mathrm{C} 17-\mathrm{C} 18-\mathrm{H} 182$ & 111.00 \\
\hline $\mathrm{C} 2-\mathrm{C} 3-\mathrm{C} 4$ & $121.2(3)$ & N19-C18-H182 & 111.00 \\
\hline $\mathrm{C} 3-\mathrm{C} 4-\mathrm{C} 5$ & $117.7(3)$ & $\mathrm{C} 17-\mathrm{C} 18-\mathrm{H} 181$ & 111.00 \\
\hline $\mathrm{C} 4-\mathrm{C} 5-\mathrm{C} 6$ & $122.1(3)$ & $\mathrm{N} 19-\mathrm{C} 18-\mathrm{H} 181$ & 111.00 \\
\hline $\mathrm{N} 9-\mathrm{C} 5-\mathrm{C} 4$ & $127.7(3)$ & $\mathrm{C} 21-\mathrm{C} 20-\mathrm{H} 201$ & 110.00 \\
\hline N9-C5-C6 & $110.1(3)$ & $\mathrm{N} 19-\mathrm{C} 20-\mathrm{H} 202$ & 110.00 \\
\hline $\mathrm{C} 5-\mathrm{C} 6-\mathrm{C} 7$ & $110.5(3)$ & $\mathrm{H} 201-\mathrm{C} 20-\mathrm{H} 202$ & 108.00 \\
\hline $\mathrm{C} 1-\mathrm{C} 6-\mathrm{C} 7$ & $129.4(3)$ & $\mathrm{N} 19-\mathrm{C} 20-\mathrm{H} 201$ & 110.00 \\
\hline $\mathrm{C} 1-\mathrm{C} 6-\mathrm{C} 5$ & $119.9(3)$ & $\mathrm{C} 21-\mathrm{C} 20-\mathrm{H} 202$ & 110.00 \\
\hline $\mathrm{C} 6-\mathrm{C} 7-\mathrm{C} 8$ & $102.5(3)$ & $\mathrm{C} 23-\mathrm{C} 22-\mathrm{H} 22$ & 118.00 \\
\hline $\mathrm{C} 16-\mathrm{C} 7-\mathrm{C} 17$ & $102.0(3)$ & $\mathrm{C} 21-\mathrm{C} 22-\mathrm{H} 22$ & 118.00 \\
\hline
\end{tabular}




\begin{tabular}{|c|c|}
\hline $\mathrm{C} 6-\mathrm{C} 7-\mathrm{C} 17$ & $112.4(3)$ \\
\hline $\mathrm{C} 8-\mathrm{C} 7-\mathrm{C} 17$ & $110.8(3)$ \\
\hline $\mathrm{C} 6-\mathrm{C} 7-\mathrm{C} 16$ & $115.4(3)$ \\
\hline $\mathrm{C} 8-\mathrm{C} 7-\mathrm{C} 16$ & $114.1(3)$ \\
\hline $\mathrm{C} 7-\mathrm{C} 8-\mathrm{C} 13$ & $116.6(3)$ \\
\hline $\mathrm{N} 9-\mathrm{C} 8-\mathrm{C} 7$ & $104.5(3)$ \\
\hline $\mathrm{N} 9-\mathrm{C} 8-\mathrm{C} 13$ & $106.0(2)$ \\
\hline $\mathrm{O} 25-\mathrm{C} 10-\mathrm{C} 11$ & $120.7(3)$ \\
\hline $\mathrm{O} 25-\mathrm{C} 10-\mathrm{N} 9$ & $122.5(3)$ \\
\hline $\mathrm{N} 9-\mathrm{C} 10-\mathrm{C} 11$ & $116.8(3)$ \\
\hline $\mathrm{C} 10-\mathrm{C} 11-\mathrm{C} 12$ & $118.1(3)$ \\
\hline $\mathrm{O} 24-\mathrm{C} 12-\mathrm{C} 11$ & $105.3(3)$ \\
\hline $\mathrm{O} 24-\mathrm{C} 12-\mathrm{C} 13$ & $114.4(3)$ \\
\hline $\mathrm{C} 11-\mathrm{C} 12-\mathrm{C} 13$ & $109.9(3)$ \\
\hline $\mathrm{C} 8-\mathrm{C} 13-\mathrm{C} 12$ & $106.8(3)$ \\
\hline $\mathrm{C} 8-\mathrm{C} 13-\mathrm{C} 14$ & $112.0(3)$ \\
\hline $\mathrm{C} 12-\mathrm{C} 13-\mathrm{C} 14$ & $117.8(3)$ \\
\hline $\mathrm{C} 13-\mathrm{C} 14-\mathrm{C} 15$ & $106.0(3)$ \\
\hline $\mathrm{C} 15-\mathrm{C} 14-\mathrm{C} 21$ & $109.8(3)$ \\
\hline $\mathrm{C} 13-\mathrm{C} 14-\mathrm{C} 21$ & $114.4(3)$ \\
\hline $\mathrm{C} 14-\mathrm{C} 15-\mathrm{C} 16$ & $108.1(3)$ \\
\hline $\mathrm{C} 7-\mathrm{C} 16-\mathrm{C} 15$ & $115.7(3)$ \\
\hline $\mathrm{N} 19-\mathrm{C} 16-\mathrm{C} 7$ & $105.0(3)$ \\
\hline $\mathrm{N} 19-\mathrm{C} 16-\mathrm{C} 15$ & $110.5(3)$ \\
\hline $\mathrm{C} 7-\mathrm{C} 17-\mathrm{C} 18$ & $103.1(3)$ \\
\hline $\mathrm{N} 19-\mathrm{C} 18-\mathrm{C} 17$ & $105.1(3)$ \\
\hline $\mathrm{N} 19-\mathrm{C} 20-\mathrm{C} 21$ & $109.7(2)$ \\
\hline $\mathrm{C} 14-\mathrm{C} 21-\mathrm{C} 20$ & $114.6(3)$ \\
\hline $\mathrm{C} 14-\mathrm{C} 21-\mathrm{C} 22$ & $123.4(3)$ \\
\hline $\mathrm{C} 20-\mathrm{C} 21-\mathrm{C} 22$ & $122.0(3)$ \\
\hline $\mathrm{C} 21-\mathrm{C} 22-\mathrm{C} 23$ & $123.3(3)$ \\
\hline $\mathrm{O} 24-\mathrm{C} 23-\mathrm{C} 22$ & $111.9(3)$ \\
\hline $\mathrm{C} 6-\mathrm{C} 1-\mathrm{H} 1$ & 120.00 \\
\hline $\mathrm{C} 2-\mathrm{C} 1-\mathrm{H} 1$ & 120.00 \\
\hline $\mathrm{C} 5-\mathrm{C} 4-\mathrm{H} 4$ & 121.00 \\
\hline $\mathrm{C} 3-\mathrm{C} 4-\mathrm{H} 4$ & 121.00 \\
\hline $\mathrm{O} 11 \mathrm{~A}-\mathrm{As} 1 \mathrm{~A}-\mathrm{C} 1 \mathrm{~A}-\mathrm{C} 2 \mathrm{~A}$ & $-51.9(3)$ \\
\hline $\mathrm{O} 11 \mathrm{~A}-\mathrm{As} 1 \mathrm{~A}-\mathrm{C} 1 \mathrm{~A}-\mathrm{C} 6 \mathrm{~A}$ & $130.0(2)$ \\
\hline $\mathrm{O} 12 \mathrm{~A}-\mathrm{As} 1 \mathrm{~A}-\mathrm{C} 1 \mathrm{~A}-\mathrm{C} 2 \mathrm{~A}$ & $73.4(3)$ \\
\hline $\mathrm{O} 12 \mathrm{~A}-\mathrm{As} 1 \mathrm{~A}-\mathrm{C} 1 \mathrm{~A}-\mathrm{C} 6 \mathrm{~A}$ & $-104.8(3)$ \\
\hline $\mathrm{O} 13 \mathrm{~A}-\mathrm{As} 1 \mathrm{~A}-\mathrm{C} 1 \mathrm{~A}-\mathrm{C} 2 \mathrm{~A}$ & $-168.3(3)$ \\
\hline $\mathrm{O} 13 \mathrm{~A}-\mathrm{As} 1 \mathrm{~A}-\mathrm{C} 1 \mathrm{~A}-\mathrm{C} 6 \mathrm{~A}$ & $13.6(3)$ \\
\hline $\mathrm{C} 25-\mathrm{O} 2-\mathrm{C} 2-\mathrm{C} 1$ & $1.0(5)$ \\
\hline $\mathrm{C} 25-\mathrm{O} 2-\mathrm{C} 2-\mathrm{C} 3$ & $-178.9(3)$ \\
\hline $\mathrm{C} 26-\mathrm{O} 3-\mathrm{C} 3-\mathrm{C} 2$ & $178.7(3)$ \\
\hline $\mathrm{C} 26-\mathrm{O} 3-\mathrm{C} 3-\mathrm{C} 4$ & $-1.1(4)$ \\
\hline $\mathrm{C} 23-\mathrm{O} 24-\mathrm{C} 12-\mathrm{C} 13$ & $-69.2(4)$ \\
\hline
\end{tabular}

$\begin{array}{ll}\mathrm{O} 24-\mathrm{C} 23-\mathrm{H} 232 & 109.00 \\ \mathrm{O} 24-\mathrm{C} 23-\mathrm{H} 231 & 109.00 \\ \mathrm{H} 231-\mathrm{C} 23-\mathrm{H} 232 & 108.00 \\ \mathrm{C} 22-\mathrm{C} 23-\mathrm{H} 232 & 109.00 \\ \mathrm{C} 22-\mathrm{C} 23-\mathrm{H} 231 & 109.00 \\ \mathrm{H} 251-\mathrm{C} 25-\mathrm{H} 253 & 109.00 \\ \mathrm{H} 252-\mathrm{C} 25-\mathrm{H} 253 & 110.00 \\ \mathrm{H} 251-\mathrm{C} 25-\mathrm{H} 252 & 109.00 \\ \mathrm{O} 2-\mathrm{C} 25-\mathrm{H} 253 & 109.00 \\ \mathrm{O} 2-\mathrm{C} 25-\mathrm{H} 251 & 110.00 \\ \mathrm{O} 2-\mathrm{C} 25-\mathrm{H} 252 & 109.00 \\ \mathrm{O} 3-\mathrm{C} 26-\mathrm{H} 261 & 110.00 \\ \mathrm{H} 261-\mathrm{C} 26-\mathrm{H} 262 & 109.00 \\ \mathrm{H} 261-\mathrm{C} 26-\mathrm{H} 263 & 109.00 \\ \mathrm{H} 262-\mathrm{C} 26-\mathrm{H} 263 & 109.00 \\ \mathrm{O} 3-\mathrm{C} 26-\mathrm{H} 262 & 110.00 \\ \mathrm{O} 3-\mathrm{C} 26-\mathrm{H} 263 & 109.00 \\ \mathrm{As} 1 \mathrm{~A}-\mathrm{C} 1 \mathrm{~A}-\mathrm{C} 2 \mathrm{~A} & 119.6(3) \\ \mathrm{C} 2 \mathrm{~A}-\mathrm{C} 1 \mathrm{~A}-\mathrm{C} 6 \mathrm{~A} & 119.0(3) \\ \mathrm{As} 1 \mathrm{~A}-\mathrm{C} 1 \mathrm{~A}-\mathrm{C} 6 \mathrm{~A} & 121.4(3) \\ \mathrm{C} 1 \mathrm{~A}-\mathrm{C} 2 \mathrm{~A}-\mathrm{C} 3 \mathrm{~A} & 119.8(4) \\ \mathrm{C} 2 \mathrm{~A}-\mathrm{C} 3 \mathrm{~A}-\mathrm{C} 4 \mathrm{~A} & 121.7(4) \\ \mathrm{N} 4 \mathrm{~A}-\mathrm{C} 4 \mathrm{~A}-\mathrm{C} 5 \mathrm{~A} & 120.9(4) \\ \text { N4A-C4A-C3A } & 121.2(4) \\ \mathrm{C} 3 \mathrm{~A}-\mathrm{C} 4 \mathrm{~A}-\mathrm{C} 5 \mathrm{~A} & 117.9(4) \\ \mathrm{C} 4 \mathrm{~A}-\mathrm{C} 5 \mathrm{~A}-\mathrm{C} 6 \mathrm{~A} & 121.1(4) \\ \mathrm{C} 1 \mathrm{~A}-\mathrm{C} 6 \mathrm{~A}-\mathrm{C} 5 \mathrm{~A} & 120.5(4) \\ \mathrm{C} 1 \mathrm{~A}-\mathrm{C} 2 \mathrm{~A}-\mathrm{H} 2 \mathrm{~A} & 120.00 \\ \mathrm{C} 3 \mathrm{~A}-\mathrm{C} 2 \mathrm{~A}-\mathrm{H} 2 \mathrm{~A} & 120.00 \\ \mathrm{C} 2 \mathrm{~A}-\mathrm{C} 3 \mathrm{~A}-\mathrm{H} 3 \mathrm{~A} & 119.00 \\ \mathrm{C} 4 \mathrm{~A}-\mathrm{C} 3 \mathrm{~A}-\mathrm{H} 3 \mathrm{~A} & 119.00 \\ \mathrm{C} 6 \mathrm{~A}-\mathrm{C} 5 \mathrm{~A}-\mathrm{H} 5 \mathrm{~A} & 119.00 \\ \mathrm{C} 4 \mathrm{~A}-\mathrm{C} 5 \mathrm{~A}-\mathrm{H} 5 \mathrm{~A} & 119.00 \\ \mathrm{C} 1 \mathrm{~A}-\mathrm{C} 6 \mathrm{~A}-\mathrm{H} 6 \mathrm{~A} & 120.00 \\ \mathrm{C} 5 \mathrm{~A}-\mathrm{C} 6 \mathrm{~A}-\mathrm{H} 6 \mathrm{~A} & 120.00 \\ & \\ & \end{array}$

$\begin{array}{ll}\mathrm{C} 17-\mathrm{C} 7-\mathrm{C} 8-\mathrm{C} 13 & -140.8(3) \\ \mathrm{C} 6-\mathrm{C} 7-\mathrm{C} 16-\mathrm{N} 19 & 153.5(3) \\ \mathrm{C} 8-\mathrm{C} 7-\mathrm{C} 16-\mathrm{N} 19 & -88.2(3) \\ \mathrm{C} 8-\mathrm{C} 7-\mathrm{C} 16-\mathrm{C} 15 & 33.9(4) \\ \mathrm{C} 17-\mathrm{C} 7-\mathrm{C} 16-\mathrm{N} 19 & 31.3(3) \\ \mathrm{C} 17-\mathrm{C} 7-\mathrm{C} 16-\mathrm{C} 15 & 153.4(3) \\ \mathrm{C} 6-\mathrm{C} 7-\mathrm{C} 17-\mathrm{C} 18 & -166.0(3) \\ \mathrm{C} 8-\mathrm{C} 7-\mathrm{C} 17-\mathrm{C} 18 & 80.1(3) \\ \mathrm{C} 6-\mathrm{C} 7-\mathrm{C} 16-\mathrm{C} 15 & -84.4(4) \\ \mathrm{C} 6-\mathrm{C} 7-\mathrm{C} 8-\mathrm{N} 9 & -17.5(3) \\ \mathrm{C} 6-\mathrm{C} 7-\mathrm{C} 8-\mathrm{C} 13 & 99.1(3)\end{array}$




\begin{tabular}{|c|c|c|c|}
\hline $\mathrm{C} 12-\mathrm{O} 24-\mathrm{C} 23-\mathrm{C} 22$ & $87.0(4)$ & $\mathrm{C} 16-\mathrm{C} 7-\mathrm{C} 8-\mathrm{N} 9$ & $-142.9(3)$ \\
\hline $\mathrm{C} 23-\mathrm{O} 24-\mathrm{C} 12-\mathrm{C} 11$ & $170.0(3)$ & $\mathrm{C} 16-\mathrm{C} 7-\mathrm{C} 8-\mathrm{C} 13$ & $-26.3(4)$ \\
\hline $\mathrm{C} 8-\mathrm{N} 9-\mathrm{C} 5-\mathrm{C} 6$ & $-3.2(4)$ & $\mathrm{C} 17-\mathrm{C} 7-\mathrm{C} 8-\mathrm{N} 9$ & $102.6(3)$ \\
\hline $\mathrm{C} 8-\mathrm{N} 9-\mathrm{C} 5-\mathrm{C} 4$ & $174.7(3)$ & $\mathrm{C} 16-\mathrm{C} 7-\mathrm{C} 17-\mathrm{C} 18$ & $-41.8(3)$ \\
\hline $\mathrm{C} 5-\mathrm{N} 9-\mathrm{C} 10-\mathrm{O} 25$ & $-24.5(5)$ & $\mathrm{N} 9-\mathrm{C} 8-\mathrm{C} 13-\mathrm{C} 12$ & $-71.7(3)$ \\
\hline $\mathrm{C} 10-\mathrm{N} 9-\mathrm{C} 5-\mathrm{C} 4$ & $22.1(6)$ & N9-C $8-\mathrm{C} 13-\mathrm{C} 14$ & $158.0(3)$ \\
\hline $\mathrm{C} 10-\mathrm{N} 9-\mathrm{C} 5-\mathrm{C} 6$ & $-155.9(3)$ & $\mathrm{C} 7-\mathrm{C} 8-\mathrm{C} 13-\mathrm{C} 14$ & $42.2(4)$ \\
\hline $\mathrm{C} 5-\mathrm{N} 9-\mathrm{C} 8-\mathrm{C} 7$ & $13.4(3)$ & $\mathrm{C} 7-\mathrm{C} 8-\mathrm{C} 13-\mathrm{C} 12$ & $172.5(3)$ \\
\hline $\mathrm{C} 5-\mathrm{N} 9-\mathrm{C} 8-\mathrm{C} 13$ & $-110.4(3)$ & $\mathrm{O} 25-\mathrm{C} 10-\mathrm{C} 11-\mathrm{C} 12$ & $150.9(4)$ \\
\hline $\mathrm{C} 10-\mathrm{N} 9-\mathrm{C} 8-\mathrm{C} 7$ & $167.6(3)$ & $\mathrm{N} 9-\mathrm{C} 10-\mathrm{C} 11-\mathrm{C} 12$ & $-29.9(5)$ \\
\hline $\mathrm{C} 10-\mathrm{N} 9-\mathrm{C} 8-\mathrm{C} 13$ & $43.9(4)$ & $\mathrm{C} 10-\mathrm{C} 11-\mathrm{C} 12-\mathrm{C} 13$ & $-0.2(4)$ \\
\hline $\mathrm{C} 8-\mathrm{N} 9-\mathrm{C} 10-\mathrm{O} 25$ & $-174.4(3)$ & $\mathrm{C} 10-\mathrm{C} 11-\mathrm{C} 12-\mathrm{O} 24$ & $123.5(3)$ \\
\hline $\mathrm{C} 8-\mathrm{N} 9-\mathrm{C} 10-\mathrm{C} 11$ & $6.3(5)$ & $\mathrm{C} 11-\mathrm{C} 12-\mathrm{C} 13-\mathrm{C} 8$ & $49.1(3)$ \\
\hline $\mathrm{C} 5-\mathrm{N} 9-\mathrm{C} 10-\mathrm{C} 11$ & $156.3(3)$ & $\mathrm{O} 24-\mathrm{C} 12-\mathrm{C} 13-\mathrm{C} 8$ & $-69.1(3)$ \\
\hline $\mathrm{C} 20-\mathrm{N} 19-\mathrm{C} 16-\mathrm{C} 15$ & $-10.7(4)$ & $\mathrm{O} 24-\mathrm{C} 12-\mathrm{C} 13-\mathrm{C} 14$ & $57.9(4)$ \\
\hline $\mathrm{C} 16-\mathrm{N} 19-\mathrm{C} 18-\mathrm{C} 17$ & $-16.7(3)$ & $\mathrm{C} 11-\mathrm{C} 12-\mathrm{C} 13-\mathrm{C} 14$ & $176.1(3)$ \\
\hline $\mathrm{C} 18-\mathrm{N} 19-\mathrm{C} 16-\mathrm{C} 7$ & $-9.6(3)$ & $\mathrm{C} 12-\mathrm{C} 13-\mathrm{C} 14-\mathrm{C} 15$ & $172.5(3)$ \\
\hline $\mathrm{C} 18-\mathrm{N} 19-\mathrm{C} 16-\mathrm{C} 15$ & $-134.9(3)$ & $\mathrm{C} 8-\mathrm{C} 13-\mathrm{C} 14-\mathrm{C} 15$ & $-63.1(3)$ \\
\hline $\mathrm{C} 20-\mathrm{N} 19-\mathrm{C} 16-\mathrm{C} 7$ & $114.7(3)$ & $\mathrm{C} 8-\mathrm{C} 13-\mathrm{C} 14-\mathrm{C} 21$ & $58.1(4)$ \\
\hline $\mathrm{C} 18-\mathrm{N} 19-\mathrm{C} 20-\mathrm{C} 21$ & $74.2(3)$ & $\mathrm{C} 12-\mathrm{C} 13-\mathrm{C} 14-\mathrm{C} 21$ & $-66.4(4)$ \\
\hline $\mathrm{C} 20-\mathrm{N} 19-\mathrm{C} 18-\mathrm{C} 17$ & $-141.3(3)$ & $\mathrm{C} 15-\mathrm{C} 14-\mathrm{C} 21-\mathrm{C} 22$ & $176.6(3)$ \\
\hline $\mathrm{C} 16-\mathrm{N} 19-\mathrm{C} 20-\mathrm{C} 21$ & $-47.3(4)$ & $\mathrm{C} 21-\mathrm{C} 14-\mathrm{C} 15-\mathrm{C} 16$ & $-54.5(3)$ \\
\hline $\mathrm{C} 2-\mathrm{C} 1-\mathrm{C} 6-\mathrm{C} 7$ & $-174.0(3)$ & $\mathrm{C} 13-\mathrm{C} 14-\mathrm{C} 15-\mathrm{C} 16$ & $69.6(3)$ \\
\hline $\mathrm{C} 6-\mathrm{C} 1-\mathrm{C} 2-\mathrm{O} 2$ & $-177.6(3)$ & $\mathrm{C} 15-\mathrm{C} 14-\mathrm{C} 21-\mathrm{C} 20$ & $-4.2(4)$ \\
\hline $\mathrm{C} 6-\mathrm{C} 1-\mathrm{C} 2-\mathrm{C} 3$ & $2.3(5)$ & $\mathrm{C} 13-\mathrm{C} 14-\mathrm{C} 21-\mathrm{C} 20$ & $-123.2(3)$ \\
\hline $\mathrm{C} 2-\mathrm{C} 1-\mathrm{C} 6-\mathrm{C} 5$ & $0.4(5)$ & $\mathrm{C} 13-\mathrm{C} 14-\mathrm{C} 21-\mathrm{C} 22$ & $57.6(5)$ \\
\hline $\mathrm{C} 1-\mathrm{C} 2-\mathrm{C} 3-\mathrm{C} 4$ & $-3.1(5)$ & $\mathrm{C} 14-\mathrm{C} 15-\mathrm{C} 16-\mathrm{N} 19$ & $62.7(3)$ \\
\hline $\mathrm{O} 2-\mathrm{C} 2-\mathrm{C} 3-\mathrm{O} 3$ & $-3.0(4)$ & $\mathrm{C} 14-\mathrm{C} 15-\mathrm{C} 16-\mathrm{C} 7$ & $-56.4(4)$ \\
\hline $\mathrm{O} 2-\mathrm{C} 2-\mathrm{C} 3-\mathrm{C} 4$ & $176.9(3)$ & $\mathrm{C} 7-\mathrm{C} 17-\mathrm{C} 18-\mathrm{N} 19$ & $36.4(3)$ \\
\hline $\mathrm{C} 1-\mathrm{C} 2-\mathrm{C} 3-\mathrm{O} 3$ & $177.1(3)$ & $\mathrm{N} 19-\mathrm{C} 20-\mathrm{C} 21-\mathrm{C} 14$ & $56.0(4)$ \\
\hline $\mathrm{O} 3-\mathrm{C} 3-\mathrm{C} 4-\mathrm{C} 5$ & $-179.2(3)$ & $\mathrm{N} 19-\mathrm{C} 20-\mathrm{C} 21-\mathrm{C} 22$ & $-124.8(4)$ \\
\hline $\mathrm{C} 2-\mathrm{C} 3-\mathrm{C} 4-\mathrm{C} 5$ & $1.0(5)$ & $\mathrm{C} 20-\mathrm{C} 21-\mathrm{C} 22-\mathrm{C} 23$ & $177.7(3)$ \\
\hline $\mathrm{C} 3-\mathrm{C} 4-\mathrm{C} 5-\mathrm{N} 9$ & $-176.0(3)$ & $\mathrm{C} 14-\mathrm{C} 21-\mathrm{C} 22-\mathrm{C} 23$ & $-3.2(5)$ \\
\hline $\mathrm{C} 3-\mathrm{C} 4-\mathrm{C} 5-\mathrm{C} 6$ & $1.8(5)$ & $\mathrm{C} 21-\mathrm{C} 22-\mathrm{C} 23-\mathrm{O} 24$ & $-62.7(5)$ \\
\hline $\mathrm{N} 9-\mathrm{C} 5-\mathrm{C} 6-\mathrm{C} 7$ & $-9.0(4)$ & $\mathrm{As} 1 \mathrm{~A}-\mathrm{C} 1 \mathrm{~A}-\mathrm{C} 2 \mathrm{~A}-\mathrm{C} 3 \mathrm{~A}$ & $-179.7(3)$ \\
\hline $\mathrm{N} 9-\mathrm{C} 5-\mathrm{C} 6-\mathrm{C} 1$ & $175.6(3)$ & $\mathrm{C} 6 \mathrm{~A}-\mathrm{C} 1 \mathrm{~A}-\mathrm{C} 2 \mathrm{~A}-\mathrm{C} 3 \mathrm{~A}$ & $-1.6(5)$ \\
\hline $\mathrm{C} 4-\mathrm{C} 5-\mathrm{C} 6-\mathrm{C} 1$ & $-2.5(6)$ & $\mathrm{As} 1 \mathrm{~A}-\mathrm{C} 1 \mathrm{~A}-\mathrm{C} 6 \mathrm{~A}-\mathrm{C} 5 \mathrm{~A}$ & $-179.2(3)$ \\
\hline $\mathrm{C} 4-\mathrm{C} 5-\mathrm{C} 6-\mathrm{C} 7$ & $172.9(3)$ & $\mathrm{C} 2 \mathrm{~A}-\mathrm{C} 1 \mathrm{~A}-\mathrm{C} 6 \mathrm{~A}-\mathrm{C} 5 \mathrm{~A}$ & $2.7(5)$ \\
\hline $\mathrm{C} 5-\mathrm{C} 6-\mathrm{C} 7-\mathrm{C} 16$ & $141.2(3)$ & $\mathrm{C} 1 \mathrm{~A}-\mathrm{C} 2 \mathrm{~A}-\mathrm{C} 3 \mathrm{~A}-\mathrm{C} 4 \mathrm{~A}$ & $-0.4(5)$ \\
\hline $\mathrm{C} 1-\mathrm{C} 6-\mathrm{C} 7-\mathrm{C} 8$ & $-168.6(4)$ & $\mathrm{C} 2 \mathrm{~A}-\mathrm{C} 3 \mathrm{~A}-\mathrm{C} 4 \mathrm{~A}-\mathrm{N} 4 \mathrm{~A}$ & $179.6(4)$ \\
\hline $\mathrm{C} 1-\mathrm{C} 6-\mathrm{C} 7-\mathrm{C} 16$ & $-44.0(5)$ & $\mathrm{C} 2 \mathrm{~A}-\mathrm{C} 3 \mathrm{~A}-\mathrm{C} 4 \mathrm{~A}-\mathrm{C} 5 \mathrm{~A}$ & $1.2(5)$ \\
\hline $\mathrm{C} 1-\mathrm{C} 6-\mathrm{C} 7-\mathrm{C} 17$ & $72.5(5)$ & $\mathrm{N} 4 \mathrm{~A}-\mathrm{C} 4 \mathrm{~A}-\mathrm{C} 5 \mathrm{~A}-\mathrm{C} 6 \mathrm{~A}$ & $-178.5(4)$ \\
\hline $\mathrm{C} 5-\mathrm{C} 6-\mathrm{C} 7-\mathrm{C} 8$ & $16.6(4)$ & $\mathrm{C} 3 \mathrm{~A}-\mathrm{C} 4 \mathrm{~A}-\mathrm{C} 5 \mathrm{~A}-\mathrm{C} 6 \mathrm{~A}$ & $-0.1(5)$ \\
\hline $\mathrm{C} 5-\mathrm{C} 6-\mathrm{C} 7-\mathrm{C} 17$ & $-102.4(4)$ & $\mathrm{C} 4 \mathrm{~A}-\mathrm{C} 5 \mathrm{~A}-\mathrm{C} 6 \mathrm{~A}-\mathrm{C} 1 \mathrm{~A}$ & $-1.8(5)$ \\
\hline
\end{tabular}


Hydrogen-bond geometry $\left(\AA,{ }^{\circ}\right)$

\begin{tabular}{lllll}
\hline$D-\mathrm{H} \cdots A$ & $D-\mathrm{H}$ & $\mathrm{H} \cdots A$ & $D \cdots A$ & $D-\mathrm{H}^{\cdots} A$ \\
\hline $\mathrm{N} 19-\mathrm{H} 19 \cdots \mathrm{O} 12 A$ & $0.91(4)$ & $1.72(4)$ & $2.610(3)$ & $168(4)$ \\
$\mathrm{N} 4 A-\mathrm{H} 41 A \cdots \mathrm{O} 4 W^{\mathrm{i}}$ & $0.89(3)$ & $2.46(4)$ & $3.291(5)$ & $155(4)$ \\
$\mathrm{N} 4 A-\mathrm{H} 42 A \cdots \mathrm{O} 3 W$ & $0.90(3)$ & $2.25(3)$ & $3.137(6)$ & $169(4)$ \\
$\mathrm{O} 13 A-\mathrm{H} 13 A \cdots \mathrm{O} 11 A^{\mathrm{ii}}$ & $0.90(4)$ & $1.67(4)$ & $2.546(3)$ & $165(4)$ \\
$\mathrm{O} 1 W-\mathrm{H} 11 W \cdots \mathrm{O} 25$ & $0.90(4)$ & $1.95(4)$ & $2.843(4)$ & $175(3)$ \\
$\mathrm{O} 1 W-\mathrm{H} 12 W \cdots \mathrm{O} 2 W^{\text {iii }}$ & $0.90(3)$ & $1.87(4)$ & $2.760(5)$ & $168(4)$ \\
$\mathrm{O} 2 W-\mathrm{H} 21 W \cdots \mathrm{O} 12 A$ & $0.90(3)$ & $2.11(3)$ & $2.945(4)$ & $153(4)$ \\
$\mathrm{O} 2 W-\mathrm{H} 22 W \cdots \mathrm{O} 11 A^{\mathrm{iv}}$ & $0.89(3)$ & $2.07(4)$ & $2.915(4)$ & $158(5)$ \\
$\mathrm{O} 3 W-\mathrm{H} 31 W \cdots \mathrm{O} 25^{\mathrm{v}}$ & $0.91(4)$ & $2.06(4)$ & $2.922(4)$ & $159(3)$ \\
$\mathrm{O} 3 W-\mathrm{H} 32 W \cdots \mathrm{O} 4 W^{\mathrm{vi}}$ & $0.91(3)$ & $1.91(3)$ & $2.791(4)$ & $164(3)$ \\
$\mathrm{O} 4 W-\mathrm{H} 41 W \cdots \mathrm{O} 1 W^{\mathrm{vii}}$ & $0.90(4)$ & $1.88(4)$ & $2.770(5)$ & $172(5)$ \\
$\mathrm{O} 4 W-\mathrm{H} 42 W \cdots \mathrm{O} 12 A$ & $0.89(4)$ & $1.91(4)$ & $2.802(4)$ & $174(5)$ \\
$\mathrm{C} 4-\mathrm{H} 4 \cdots \mathrm{O} 25$ & 0.95 & 2.37 & $2.900(4)$ & 115 \\
$\mathrm{C} 6 A-\mathrm{H} 6 A \cdots \mathrm{O} 13 A$ & 0.95 & 2.55 & $3.011(4)$ & 110 \\
$\mathrm{C} 8-\mathrm{H} 8 \cdots \mathrm{O} 24$ & 1.00 & 2.60 & $3.009(4)$ & 104 \\
$\mathrm{C} 14-\mathrm{H} 14 \cdots \mathrm{O} 33^{\mathrm{viii}}$ & 1.00 & 2.52 & $3.363(4)$ & 142 \\
$\mathrm{C} 15-\mathrm{H} 151 \cdots \mathrm{O} 11 A^{\mathrm{ii}}$ & 0.99 & 2.60 & $3.561(4)$ & 165 \\
$\mathrm{C} 18-\mathrm{H} 182 \cdots \mathrm{O} 2 W$ & 0.99 & 2.58 & $3.422(5)$ & 143 \\
$\mathrm{C} 20-\mathrm{H} 201 \cdots \mathrm{O} 11 A^{\mathrm{ii}}$ & 0.99 & 2.41 & $3.388(4)$ & 170 \\
$\mathrm{C} 20-\mathrm{H} 202 \cdots \mathrm{O} 13 A^{\text {iv }}$ & 0.99 & 2.43 & $3.229(4)$ & 137 \\
& & & & \\
\hline
\end{tabular}

Symmetry codes: (i) $x-1 / 2,-y+3 / 2,-z+1$; (ii) $x+1 / 2,-y+1 / 2,-z+1$; (iii) $-x, y-1 / 2,-z+1 / 2$; (iv) $x-1 / 2,-y+1 / 2,-z+1$; (v) $-x+1 / 2,-y+1, z+1 / 2$; (vi) $x+1 / 2$, $-y+3 / 2,-z+1 ;$ (vii) $-x+1, y+1 / 2,-z+1 / 2 ;$ (viii) $-x+1, y-1 / 2,-z+1 / 2$. 